\title{
COMETARY PLASMA WAVES AND INSTABILITIES
}

\author{
BRUCE T. TSURUTANI \\ Jet Propulsion Laboratory \\ California Institute of Technology \\ 4800 Oak Grove Drive \\ Pasadena, California 91109
}

\begin{abstract}
This review will discuss various plasma waves and instabilities that have been observed near comets. Comments on nonlinear wave evolution and wave cascading, as well as the role of nonlinear waves in wave-particle interactions, will be made.
\end{abstract}

\section{Introduction}

The International Cometary Explorer (ICE) mission to Comet Giacobini-Zinner and the spacecraft "armada" (Suisei, Sakigake, VEGAs 1 and 2, Giotto, and ICE) to Comet Halley have given us our first in situ measurements at comets (see Science, April 1986; Geophys. Res. Lett., March-April 1986; Nature, May 1986; ESA SP-250, December 1986; Astron. Astrophys., 187, 1987). Because of the long ionization times for cometary neutrals, the ions extend to millions of kilometers from the nucleus. The interaction of these ions and electrons with the solar wind has provided us with a unique laboratory to study plasma waves and instabilities. In this review, I will first attempt to order observational results by plasma parameters and compare them with specific instabilities. Although it is not possible to control the space plasma "laboratory," the variation in the cometary ion and electron beam densities and velocities can be studied by examining regions far from and near to the comet nucleus. Similarly, we cannot control the initial pitch angle of the pickup ions, but by using directional discontinuities naturally provided to us by the solar wind, intervals of a variety of particle pitch angles are available. Thus, by resourcefulness, a variety of plasma parameters can be made available ( $\mathrm{N}$ beam, $\rho$, etc.). Clearly, the entire available parameter space has not been fully examined. It has been partially studied, and the results obtained to date will be reviewed.

Cometary plasma wave research has also allowed us to study several new topics that are just beginning to unfold: nonlinear wave evolution, wave cascading, and the development of turbulence. The second section of this review will discuss spacecraft observations relevant to these topics. I will also briefly comment on the implications of the results for wave-particle interactions: linear, nonlinear, resonant and nonresonant, and their effects on stochastic particle acceleration.

In the third section, I will review the higher frequency ELF/VLF (Extremely Low Frequency/Very Low Frequency) wave observations. Only four spacecraft were equipped 
with instrumentation that could make such measurements: ICE, Sakigake, and VEGAs 1 and 2. Comparisons between the various measurements will be made.

\section{Low-Frequency Plasma Waves}

\subsection{THEORETICAL BACKGROUND}

The initial ion pickup pitch angle depends on the orientation of the interplanetary magnetic field (IMF) relative to the solar wind velocity. I will call this angle $\alpha$. This orientation determines much of the ion distribution function and hence the specific instability that leads to wave growth and pitch angle scattering. Two extreme examples are illustrated for tutorial purposes. These are given in Figures 1 and 2.

Figure 1 illustrates the case where the ambient IMF is aligned orthogonally to the solar wind, $\mathrm{V}_{\mathrm{sw}}$. The $\vec{V}_{s w} \times \vec{B}$ motional electric field acts on the newly created cometary ions to accelerate them into a cycloidal motion (in the inertial frame). The cometary ion will have both zero velocity and twice the solar wind velocity during its motion. It is the latter case that allows energetic particle detectors to measure the ion fluxes and distributions (Hynds et ai., 1986; Ipavich et al., 1986; Somogyi et al., 1986; Mukai et al. 1986; Balsiger et al., 1986; Korth et al., 1986). With a typical solar wind velocity of $400 \mathrm{~km} / \mathrm{s}$, the peak kinetic energy of a cometary water group ion is $1 / 2 \mathrm{~m}\left(2 \mathrm{~V}_{\mathrm{sw}}\right)^{2} \approx 60 \mathrm{keV}$.

The ion motion is more easily visualized in the solar wind (plasma) frame. The ions gyrate about the magnetic field with a velocity $\mathrm{V}_{\mathrm{sw}}$. The ions are also convected past the comet and spacecraft with a velocity $\mathrm{V}_{\mathrm{sw}}$.

The above ion anisotropy can lead to three low-frequency instabilities: the ion cy-

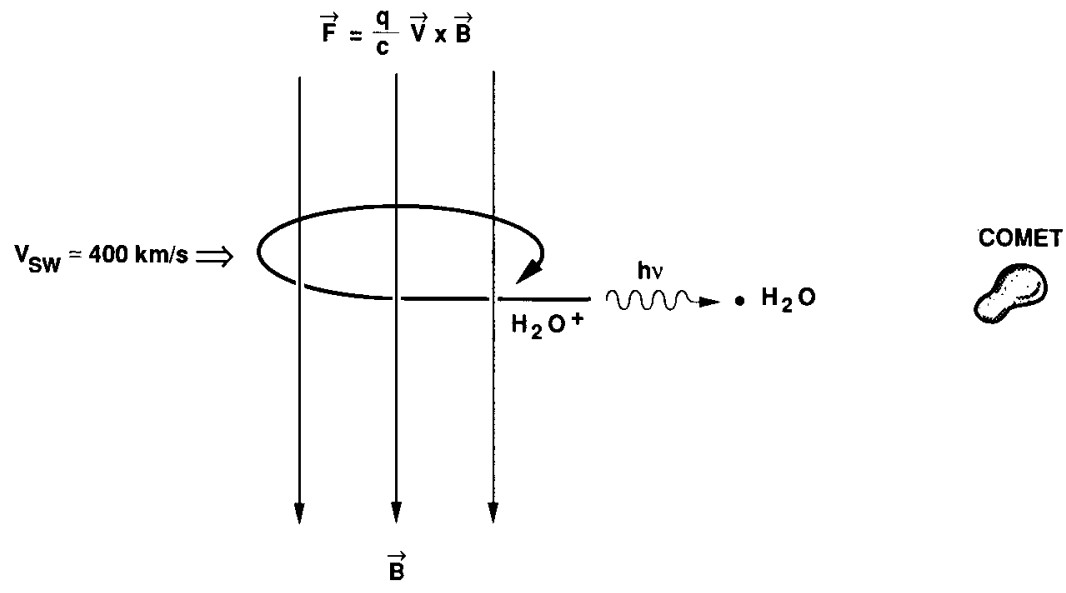

Figure 1. A schematic illustrating cometary ion pickup when the interplanetary magnetic field (IMF) is perpendicular to the solar wind velocity vector. In the plasma frame, ions form a ring distribution with velocity $\mathrm{V}_{\mathrm{SW}}$ relative to the magnetic field. 


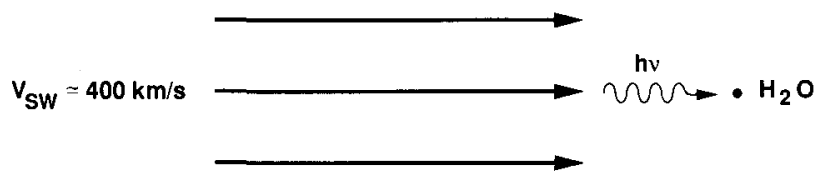

Figure 2. An example illustrating cometary ions when the IMF is parallel to the solar wind velocity vector. There is no $\vec{V}_{s w} \times \vec{B}$ motional force. However, the ions do form a beam of velocity $-\vec{V}_{s w}$ in the plasma frame.

clotron instability (Kennel and Petschek, 1966; Thorne and Tsurutani, 1987; Brinca and Tsurutani, 1988a; Lee, 1989; Gary, 1990), a parallel propagating nonoscillatory mode (Brinca and Tsurutani, 1987a, b), and a fluid mirror instability (Chandrasekhar et al., 1958; Hasegawa, 1969, Price et al., 1986; Lee et al., 1987; Price, 1989; Hasegawa and Chen, 1989). These various modes can be easily distinguished experimentally. Assuming that the angle $\alpha$ is not exactly $90^{\circ}$ and that there is a parallel component of the ion velocity along the magnetic field with $V_{\|}>V_{A}$, where $V_{A}$ is the Alfvén speed, the ion cyclotron instability leads to resonant left-hand waves propagating along $\vec{B}$ in the opposite direction as the ions.

The mirror mode is a nonoscillatory mode. The instability conditions for this are:

$$
\frac{\beta_{\perp}}{\beta_{\|}}>1+1 / \beta_{\perp}
$$

where $\beta$ is the ratio of the plasma to magnetic pressure (Crooker and Siscoe, 1977). Thus, this fluid instability occurs for cases of large anisotropies or in high $\beta$ regions such as the magnetosheath. The mirror mode is most easily recognized by the large magnetic field magnitude variations and small directional changes (see Price et al., 1986 and Lee et al., 1987 for numerical simulation illustrations). The latter is caused by the dominance of growth of this mode at large angles to $\vec{B}_{\mathrm{o}}\left(60^{\circ}\right.$ to $80^{\circ}$; Price et al., 1986). An example of this mode from a computer simulation is shown in Figure 3, taken from Price et al. (1986).

It should be noted that although the above picture is generally accurate, it is not totally correct. There are additional (small) ion velocities that must also be considered under certain situations. In most cases they are negligible. The cometary neutrals have velocities radially away from the nucleus. The velocity is $\sim 1 \mathrm{~km} / \mathrm{s}$ for the case of $\mathrm{H}_{2} \mathrm{O}$ molecules and $\sim 8$ and $20 \mathrm{~km} / \mathrm{s}$ for the case of hydrogen atoms. There are also thermal velocities associated with the atoms and molecules that are less than the above streaming velocities. Finally, there are small velocities associated with photoionization and charge exchange processes. These velocities are small in comparison to solar wind speeds and can be neglected for most calculations. However, because of the presence of these small velocities (and because the IMF has directional fluctuations), it is clear that the ion pickup distribution is not 

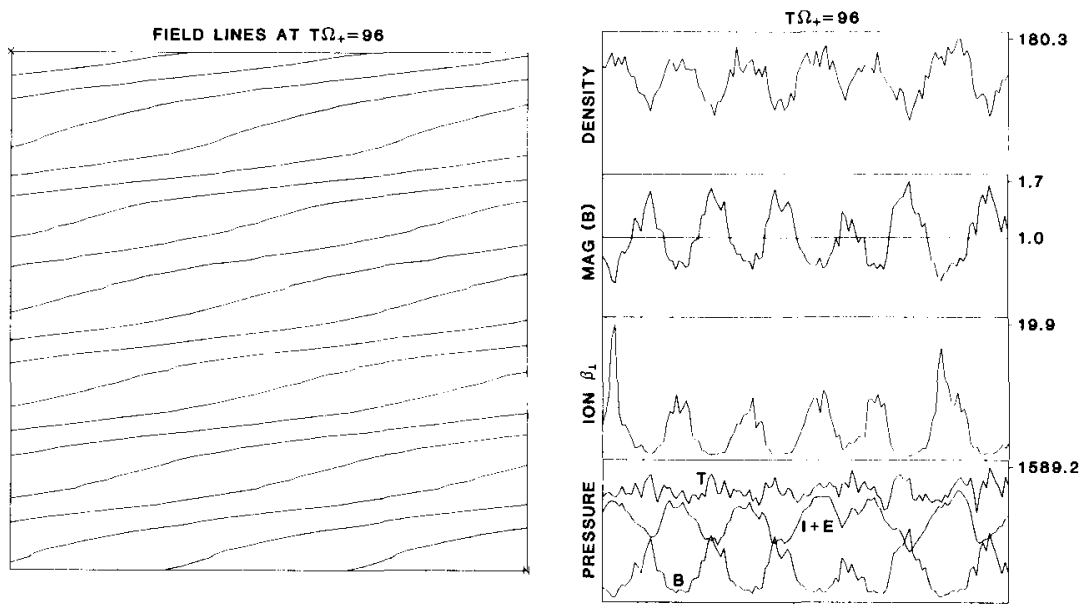

Figure 3. The density, magnetic field magnitude, perpendicular ion plasma beta, and perpendicular pressures from a 1-D simulation. The wave normal angle is $80^{\circ}$. B corresponds to the magnetic pressure, $\mathrm{I}+\mathrm{E}$, the plasma pressure and $\mathrm{T}$, the total pressures. The structures are time-stationary. Note the anticorrelation between the density and field magnitude, the large variations in $\beta$, and the near-constant total pressure.

a pure delta function, as has been idealized for some calculations (see Lee, 1989, for discussion).

Figure 2 illustrates the case where the ambient magnetic field is parallel to the solar wind velocity vector. In this instance, there is no $\vec{V}_{s w} \times \vec{B}$ solar wind force. On the other hand, the ions form a beam in the solar wind (plasma) frame moving at a velocity $-\vec{V}_{s w}$ relative to the ambient plasma. Two types of instabilities can result from this distribution: a right-hand resonant helical (ring) beam instability and a fluid, nonresonant instability.

The right-hand resonant helical beam instability can have strong wave growth, not only for a pure beam $\left(\alpha=0^{\circ}\right)$, but also for angles up to $\alpha \approx 70^{\circ}$. This instability is fed by the free energy associated with both the gyro motion energy $\left(E_{\perp}\right)$ and the energy parallel to the field $\left(\mathrm{E}_{\|}\right)$. This was first discussed by $\mathrm{Wu}$ and Davidson (1972) and $\mathrm{Wu}$ and Hartle (1974) for the pickup of interstellar and planetary neutrals. A large amount of theoretical work took place after the comet encounters. Recent results are found in Winske et al. (1985), Winske and Gary (1986), Sharma and Patel (1986), Thorne and Tsurutani (1987), Goldstein and Wong, (1987), Lee and Ip (1987), Brinca and Tsurutani (1988a), Gary and Madland (1988) and Price et al. (1988). Recent summaries of the theoretical work can be found in Lee (1989) and Gary (1990). Pure beam instabilities (the case shown in Figure 2) were originally discussed in Fairfield (1969) and Barnes (1979). The conditions for cyclotron resonance is the standard relationship $\omega=\vec{k} \cdot V+n \Omega_{i}$, where $\omega$ is the wave frequency, $\vec{k}$ and $\vec{V}$ the wave $\mathrm{k}$ vector and particle velocity, $\mathrm{n}$ an integer, and $\Omega_{\mathrm{i}}$ the ion gyrofrequency. For this instability, $\vec{k}$ and $\vec{V}$ have the same directional sense. The nonresonant or firehose instability is a fluid instability that grows when the parallel pressure is large 
in comparison to the perpendicular plus magnetic pressure. The plasma is unstable when $\mathrm{P}_{\| \mid}>\mathrm{P}_{\perp}+\mathrm{B}^{2} / 4 \pi$. This has close analogies to the instability derived from water squirting from a hose; hence, the name. Past works in this area can be found in Sagdeev and Vedenov (1958), Sentman et al. (1981), Gary et al. (1984), Winske and LeRoy (1984), Sagdeev et al. (1986), and Galeev et al. (1990). Gary et al. (1985) and Gary (1990) have shown that this wave mode has sufficient growth only for beams with very high velocities and/or densities. For proton beams, a number density of $\sim 1 \%$ and a beam velocity $>10$ to $15 \mathrm{~V}_{\mathrm{A}}$ are necessary for wave growth.

These above two wave modes can be easily identified. In the right-hand resonant instability, the ion beam overtakes the waves, so there is an anomalous Doppler shift in the ion frame of reference (the ion senses the waves as left-hand polarized, rotating in the same sense as its gyromotion about the ambient magnetic field). Since the ions form a beam in the solar wind frame, the ions and the generated waves propagate in the direction opposite to the solar wind direction, or towards the Sun. For waves propagating parallel to the ambient magnetic field, the phase velocity is substantially less than the solar wind speed (with the exception of regions very close to the comet) and the waves will be anomalously Doppler shifted in the spacecraft frame. These waves will be observed as left-hand polarized.

The nonresonant instability generates low-frequency left-hand waves propagating counter to the ion beam. To distinguish these waves from the anomalously Doppler shifted right-hand waves discussed above, we note that the waves are not necessarily generated at resonant frequencies. Because the spacecraft velocities relative to the comet were small in comparison to the solar wind speed ( $21 \mathrm{~km} / \mathrm{s}$ in the case of ICE at Giacobini-Zinner and 65 to $70 \mathrm{~km} / \mathrm{s}$ for the armada at Halley), one can, to the first order, view the spacecraft as being in the cometary ion frame. Thus waves generated by resonant instabilities were measured at the cometary ion cyclotron frequency (Tsurutani and Smith, 1986a, 1986b; Saito et al., 1986; Johnstone et al., 1987a,1987b; Glassmeier et al., 1987). The nonresonant waves would be detected at lower frequencies. No concrete evidence for the presence of the latter waves has been obtained yet.

\subsection{OBSERVATIONAL RESULTS}

2.2.1. Right-Hand Resonant Helical Beam Instability. Figure 4 illustrates the presence of waves at Giacobini-Zinner near the $\mathrm{H}_{2} \mathrm{O}$ group (16 to $19 \mathrm{amu}$ ) ion cyclotron frequency. The field components are in Geocentric Solar Ecliptic (GSE) coordinates. In this coordinate system, the $\hat{x}$-axis is towards the Sun, $\hat{y}$ is in the plane of the ecliptic in the direction opposite to that of planetary rotation, and $\hat{z}$ corresponds to the north ecliptic pole. A spectrum of an "active" solar wind has been taken from Siscoe et al. (1968) and is superposed on the figure's three panels. It is noted that the peak in the three power spectra occurs near 100 seconds, the $\mathrm{H}_{2} \mathrm{O}$ group ion cyclotron frequency for this interval.

Johnstone et al. (1986) examined power spectra of the solar wind proton velocity components (in field-aligned coordinates) at Comet Halley, and found a similar peak at the $\mathrm{H}_{2} \mathrm{O}$ group ion cyclotron frequency. The velocity deviations are up to $10 \mathrm{~km} / \mathrm{s}$, and the largest amplitudes are in the two transverse components. In the examples given by Glassmeier et al. (1987), the magnetic field deviations are typically $\Delta \vec{B} / \mathrm{B} \approx 0.5$ and $\Delta|\mathrm{B}| \mathrm{B} \approx 0.1$ for Halley. 

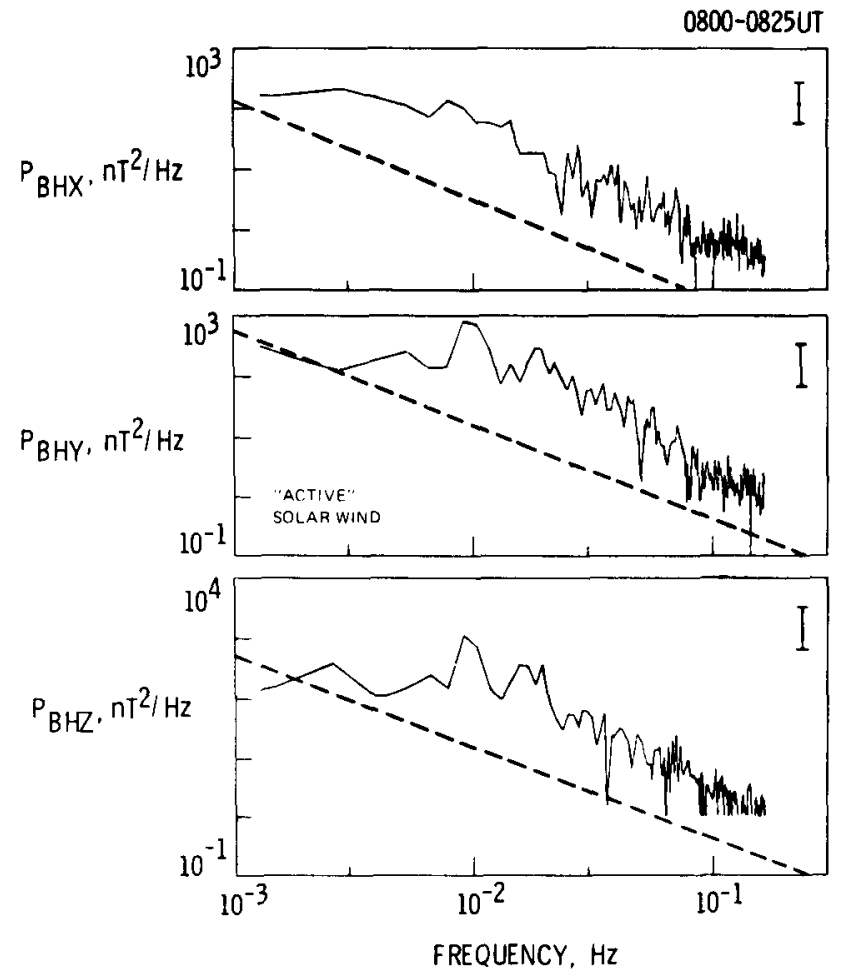

Figure 4. Power spectra of the (GSE) magnetic field components of Comet GiacobiniZinner. A power law curve, representative of an active solar wind, is also shown for comparative purposes. The wave intensities are well above solar wind fluctuation levels. The waves are considerably more intense than those at Halley. From Tsurutani and Smith (1986b).

The wave fluctuation intensities at Giacobini-Zinner were considerably higher than during the flyby of Comet Halley. Not only were the wave intensities greater, but the wave forms were highly steepened as well (shown later). The peak-to-peak $\Delta \vec{B} / \mathrm{B}$ at GiacobiniZinner was $\approx 2.0$, and $\Delta \mathrm{B} \mid \mathrm{B} \approx 0.5$. It is thought that the smaller neutral production of comet Giacobini-Zinner, $\mathrm{Q}=2 \times 10^{28}$ molecules $/ \mathrm{s}\left(\mathrm{Q}_{\mathrm{GZ}} \approx \mathrm{Q}_{\mathrm{H}} / 35\right)$, led to a shock formation at an upstream distance ten times closer to the nucleus $\left(10^{5} \mathrm{~km}\right.$, instead of the $10^{6} \mathrm{~km}$ for the case of Halley), and this closeness is related to the stronger waves at Giacobini-Zinner (V. Shapiro, personal communication, 1989).

Figure 5 illustrates the spatial extent of the waves in the cometary (GiacobiniZinner) environment. Each point represents a 25 -minute average power spectral density. The left-hand panel is for transverse fluctuations, and the right-hand panel (x-axis) indicates compressional fluctuations. With a relative spacecraft-comet velocity of $21 \mathrm{~km} / \mathrm{s}$, each day represents a distance of $\sim 1.8 \times 10^{6} \mathrm{~km}$. Thus, from the figures, it is apparent 


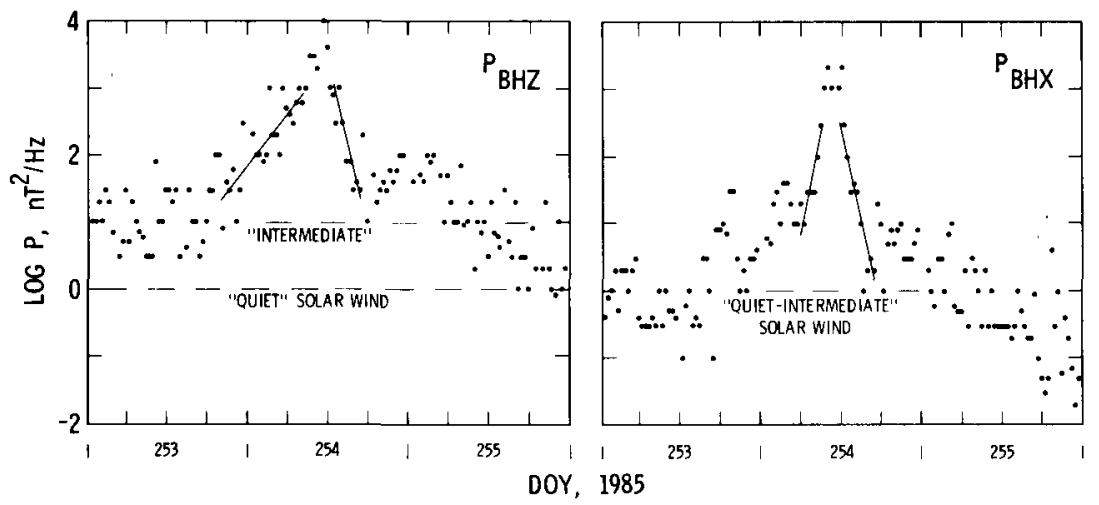

Figure 5. Twenty-five-minute average power spectral densities at $10^{2} \mathrm{~s}$, the $\mathrm{H}_{2} \mathrm{O}$ group ion cyclotron frequency. The $\mathrm{x}$-axis is aligned along the ambient field, and $\mathrm{z}$ is transverse to the field. Thus, right-hand and left-hand panels represent compressional and transverse fluctuations, respectively. Typical interplanetary fluctuation intensities are shown for comparison. From Tsurutani and Smith (1986b).

that the cometary influence extends at least one million kilometers from the comet nucleus and perhaps further. These wave amplitudes have been modelled by Gary et al. (1988) and have been shown to fit quasilinear predictions, assuming the previously given neutral production rate, a neutral velocity of $1 \mathrm{~km} / \mathrm{s}$, and an ionization rate of $10^{6} \mathrm{~s}$. In comparison, the Very Low Frequency (VLF) electrostatic component of the plasma waves has been detected to a distance at least as far as $2 \times 10^{6}$ to $3 \times 10^{6} \mathrm{~km}$ and pickup ions as far as $8 \times 10^{6} \mathrm{~km}$. It is possible that the ICE instrumentation detected cometary effects as far as $28 \times 10^{6} \mathrm{~km}$ from Halley (Scarf et al., 1986a; Wenzel et al., 1986), but the neutral atoms/molecules would have had to have very high velocities to be able to reach such large distances. These results remain quite controversial, with Daly (1987) and Tsurutani et al. (1987a) giving arguments against this interpretation.

Using both the solar wind proton and magnetic field data, Glassmeier et al. (1989) have obtained cross spectral densities of the plasma (shown in Figure 6). This figure was taken from an interval where the magnetic field was nearly aligned with the solar wind velocity and is given in a Halley-Centered Solar Ecliptic (HSE) coordinate system where the $x$-axis is directed from Halley towards the Sun and the $y$-axis is opposite to the direction of planetary orbital motion. The z-axis forms a right-hand system. This particular plot shows the magnetic field $z$-component. The top panel illustrates the presence of several peaks, with the cyclotron fundamental at $7 \mathrm{mHz}$ and harmonics at 14,21,29 and $35 \mathrm{mHz}$. Note that the coherency is $>0.9$ for all of these peaks (shown in the middle panel). Glassmeier et al. (1989) stated that the other two components were studied, and similar results were obtained.

The waves at $7 \mathrm{mHz}$ are linearly polarized and the higher order harmonic waves are either linearly or highly elliptically polarized. Theoretical discussion concerning the gen- 


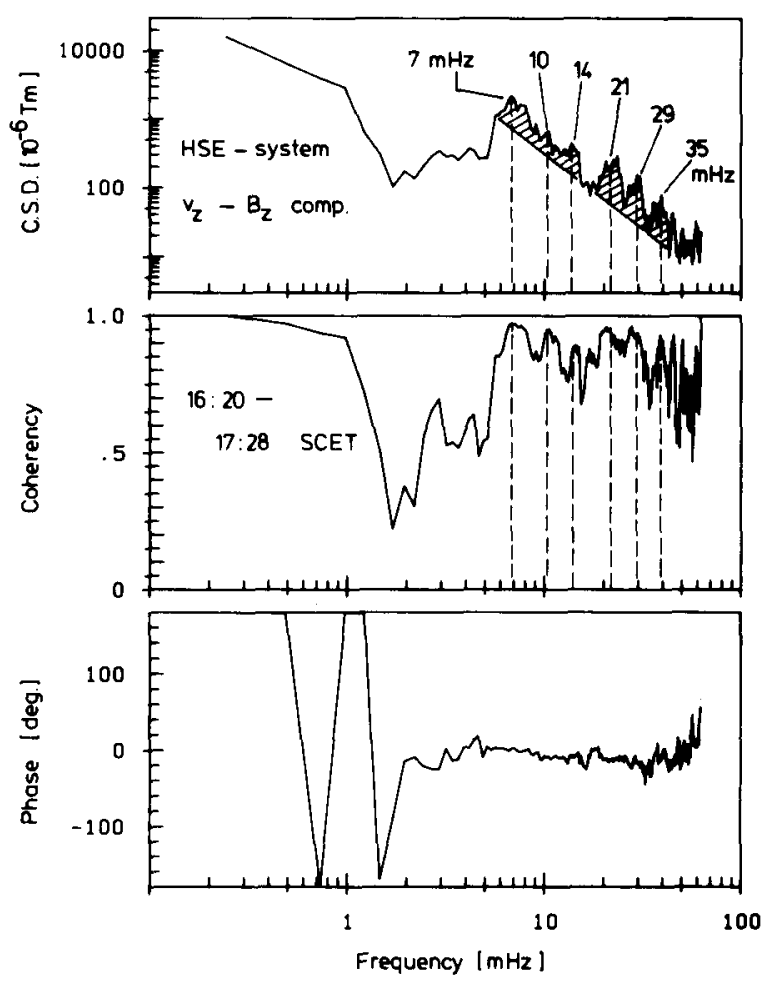

Figure 6. From top to bottom, the cross-spectral densities of the solar wind proton velocity and magnetic field, the coherency, and the phase relationship between velocity and $\vec{B}$. The interval analyzed occurred when the IMF $\vec{B}$ was along the solar wind velocity direction. Several significant power spectral density peaks are: the $\mathrm{H}_{2} \mathrm{O}$ group ion cyclotron frequency at $7 \mathrm{mHz}$, and cyclotron harmonics at 14,21,29 and $35 \mathrm{mHz}$. From Glassmeier et al. (1989).

eration of these waves can be found in Smith et al. (1985), Smith and Gary (1987), Brinca and Tsurutani (1989), and Goldstein et al. (1990).

2.2.2. Waves Detected Far Upstream of the Cometary Bow Shock. Waves were examined at a distance up to $7 \times 10^{5} \mathrm{~km}$ from Comet Giacobini-Zinner $(\sim 7$ times the bow shock stand-off distance). Several examples are shown in Figures 7 and 8 . A variety of polarizations have been detected, from circularly polarized waves propagating at large angles relative to the ambient field $\left(\theta_{\mathrm{kB}}=29^{\circ}\right.$ in Figure 7$)$ to highly elliptically polarized waves $\left(\lambda_{1} / \lambda_{2}=11\right)$ propagating at only $19^{\circ}$ relative to $\vec{B}$ (Figure 8 ). Even at these large distances, the wave transverse components were typically $\Delta \vec{B} / \mathrm{B} \sim 0.5$, with a compressional component $\Delta \mathrm{B} B \mathrm{~B} / \mathrm{B} \sim 0.1$ to 0.3 . 


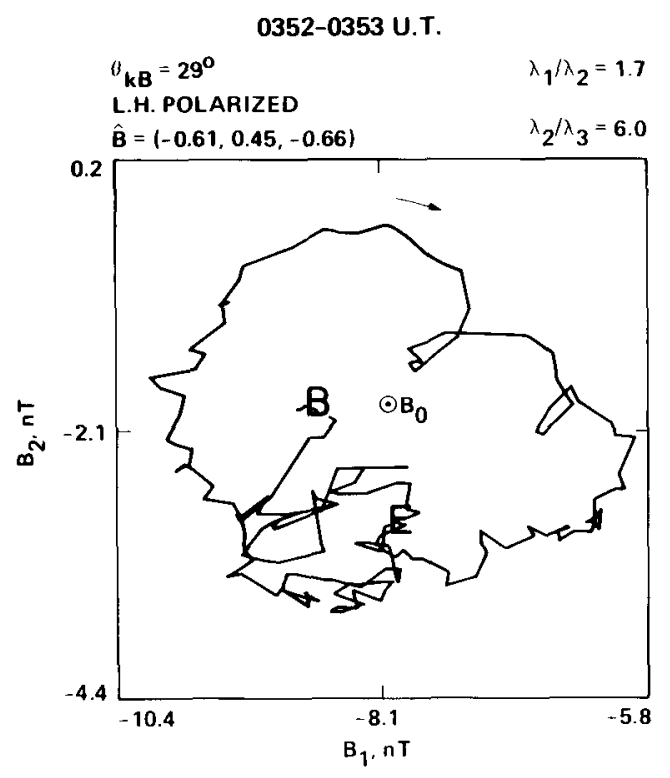

Figure 7. Hodograms for a wave detected at $\sim 5 \times 10^{5} \mathrm{~km}$ upstream of Comet Giacobini-Zinner. The standard notation is used: $\mathrm{B}_{1}, \mathrm{~B}_{2}, \mathrm{~B}_{3}$ correspond to the magnetic field in the maximum, intermediate, and minimum variance directions. The wave is lefthand circularly polarized in the spacecraft frame and is propagating at an angle of $29^{\circ}$ relative to $\vec{B}$. The ambient magnetic field direction $\hat{B}$ is given for reference. In this case, $\alpha=52^{\circ}$, typical of a Parker spiral, except with a large southward component. From Tsurutani et al. (1989a).

The wide variety of polarization and direction of propagation of the waves has not been adequately explained by theory. Linear theory predicts the growth of parallel propagating, noncompressive waves. Very few waves with the latter properties have been detected to date.

2.2.3. Waves at Comet Halley. The waves detected upstream of Comet Halley's bow shock, although smaller in amplitude than those near Comet Giacobini-Zinner's bow shock, were less ordered and much more turbulent in appearance. An example of the waves taken from $2 \times 10^{6}$ to $1.75 \times 10^{6} \mathrm{~km}$ from the nucleus is given in Figure 9 (from Johnstone et al., 1986).

Figure 9 is the proton data taken from the plasma experiment (Johnstone et al., 1976). The wave compressional components are small (the y component is nearly constant). It is noted that at times, the fluctuations appear relatively ordered, and at other times, relatively more disordered. At the beginning and end of this interval, there are short durations where the field and plasma velocities are somewhat correlated.

Figure 10 gives the hodograms for the transverse plasma components (right-hand panels) for these "correlated" intervals. The magnetic field hodograms are shown in the 
0421:14-0422:16 U.T.

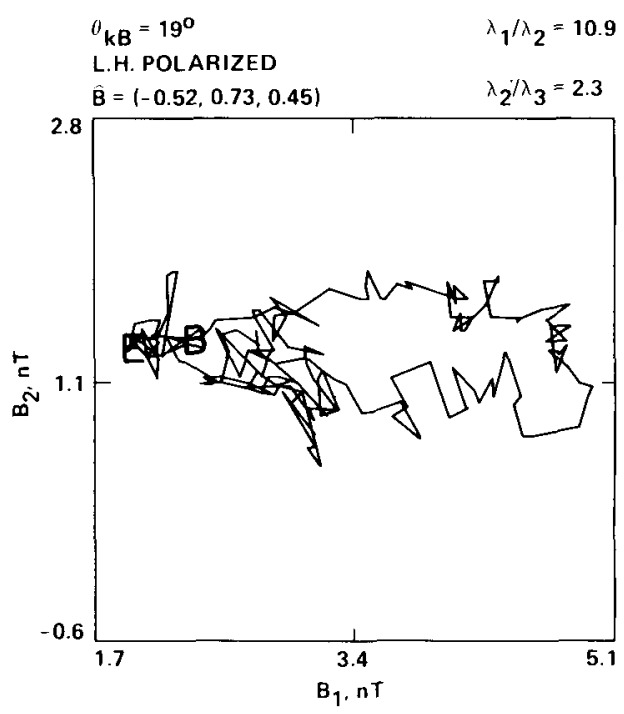

Figure 8. Similar to Figure 7. The wave is highly elliptically linearly polarized $\left(\lambda_{1} / \lambda_{2}=10.9\right)$ and is propagating at an angle $19^{\circ}$ relative to $\vec{B}$. From Tsurutani et al. (1989a).

left-hand panels. It is clear from both the plasma and field hodograms that there is no dominant wave polarization. The results give "a good impression that linearly polarized waves are present" (Johnstone et al., 1986).

2.2.4. Resonant L-Mode Waves? $\alpha=90^{\circ}$ Intervals Far From the Comet. When $\alpha$ is close to $90^{\circ}$, the wave mode expected is the left-hand ion cyclotron wave (and harmonics). Such waves have been searched for using the occurrence of interplanetary discontinuities. At large upstream distances, the anisotropic pressure associated with the pickup cometary ions is insufficient for the growth of the mirror mode (this can be easily calculated from the expression given previously).

An example of this solar wind condition is given in Figure 11, taken from an interval some $5.5 \times 10^{5} \mathrm{~km}$ upstream of Giacobini-Zinner. This figure illustrates that both prior to and after the interval where $\alpha \approx 90^{\circ}$ (shaded interval), heavy $\left(\mathrm{H}_{2} \mathrm{O}\right.$ group) ion cyclotron waves are present. However, during the interval when $\alpha \approx 90^{\circ}$, there is an absence of any long-period $\left(\mathrm{H}_{2} \mathrm{O}\right.$ group $)$ ion waves. Other intervals of this type have been studied, with similar results.

Although Gary et al. (1989) predicted decreasing wave amplitude with increasing $\alpha$, the total lack of measurable left-hand waves at Comet Giacobini-Zinner is presently somewhat of a mystery. Although such specific relationships have not been examined at Comet Halley, it is noted that Glassmeier et al. (1989) found a lack of a specific peak at the 


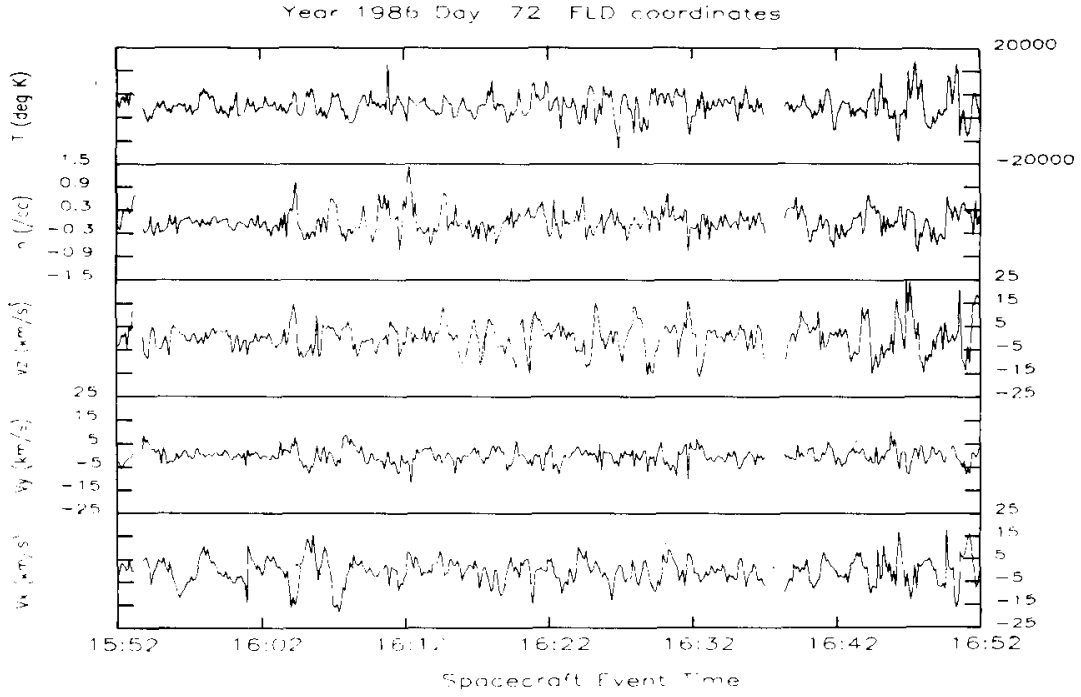

Figure 9. Example of waves in the solar wind proton data taken from $1.75 \times 10^{6}$ to $2.0 \times 10^{6} \mathrm{~km}$ from Halley (for reference, the Halley shock occurred at $1.1 \times 10^{6} \mathrm{~km}$ ). From top to bottom are the proton temperature, density, and the velocity components. In this coordinate system, the y-axis is parallel to $\vec{B}, \hat{z}$ is parallel to $-\vec{V}_{s w} \times \vec{B}$, and the $\mathrm{x}-$ axis forms a right-hand system. The wave compressional component is quite small. The fluctuations are, in general, relatively disordered. From Johnstone et al. (1986).

$\mathrm{H}_{2} \mathrm{O}$ group ion cyclotron frequency when $\alpha$ was near $90^{\circ}$. The total wave power was also considerably less. An example of this will be shown later in this review.

Figure 12 gives plots of the logarithm of the flux of the water group ions and the sine of the angle $\alpha$ versus time. It is noted that when $\alpha$ is large, there are enhanced fluxes of $65-$ to $95-\mathrm{keV}$ ions detected (vertical shaded regions). Such features have previously been discussed by Sanderson et al. (1986). This figure includes the interval shown in Figure 11. A detailed examination of the pitch angle anisotropy using three energetic ion detectors (Hynds et al., 1986) indicates that there is little, if any, pitch angle scattering during the high- $\alpha$ interval (Tsurutani et al., 1989a). More recent work on ion anisotropies by Richardson et al. (1989a) has given results that are in good agreement with the above. These conclusions are in accord with the lack of left-hand waves to provide the scattering.

It is relevant to point out that the lack of left-hand waves has also been noted in the Earth's magnetosheath. Price et al. (1986) have performed 1-D simulations and found a higher linear growth rate for the left-hand cyclotron mode than for the mirror mode. Yet in magnetosheath plasmas at the Earth, Jupiter, and Saturn, the mirror mode is typically the most obvious wave mode in the data (Tsurutani et al., 1982). There is no accepted explanation at this time. Price et al. (1986) and Hada (1986) have suggested that the presence of minor ions species could decrease the growth of the ion cyclotron waves (but not influence 

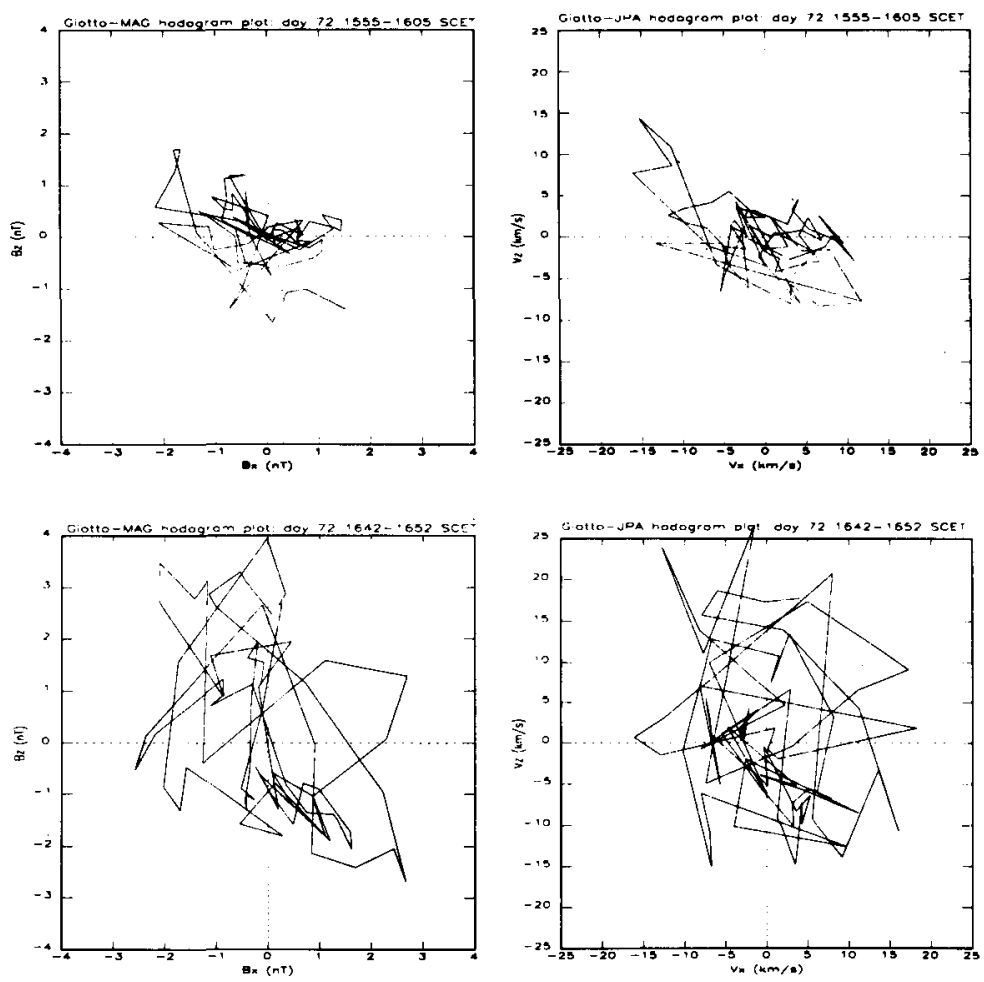

Figure 10. Hodograms for the transverse components $(\hat{z}, \hat{x})$ of the magnetic field (left). and the proton velocity (right). Two time intervals where $\vec{B}$ and $\vec{V}$ are "well correlated" are illustrated. There is no apparent dominant wave polarization present. From Johnstone et al. (1986).

the mirror mode growth rate), but the percent density necessary to accomplish this is unreasonably high (Hada, 1986).

One possible suggestion is that long convective growth times are necessary for lefthand waves to attain measurable amplitudes (Tsurutani et al., 1989a). This implies steady ambient magnetic fields, as exist in the magnetosphere during the recovery phase of magnetic storms (Erlandson et al., 1989). However, the fluctuating fields typically present in the magnetosheath and in a cometary environment may cause substantial wave damping, inhibiting significant growth. It is suggested that a simple computer simulation using first quiet and then turbulent ambient fields might be useful to answer this question.

The observations leave another unanswered question: how do the cometary ions become isotropized when $\alpha \approx 90^{\circ}$, if they do at all? Perhaps there are Extremely Low Frequency (ELF) and Very Low Frequency (VLF) electrostatic waves that are present during such conditions. A search for possible modes should be undertaken and, if found, the pitch angle scattering rates calculated and compared with the observations. 

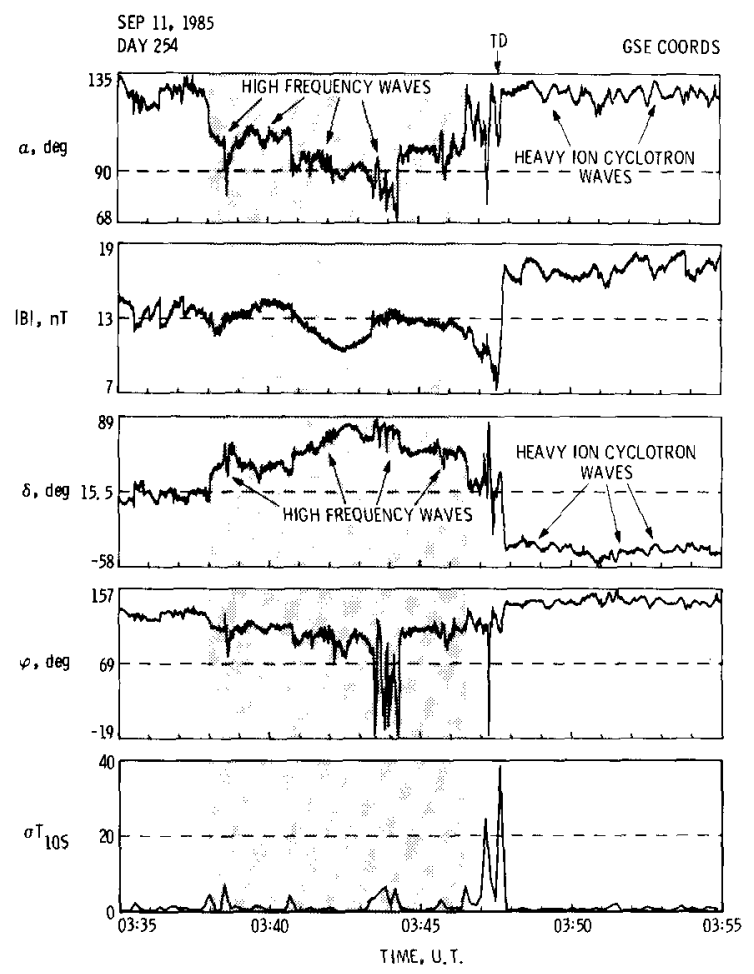

Figure 11. An interval (shaded region) where $\alpha$ is close to $90^{\circ}$. There is an absence of waves with frequencies near the $\mathrm{H}_{2} \mathrm{O}$ group ion cyclotron frequency. From Tsurutani et al. (1989b).

One surprising result was the detection of short-duration magnetic pulses during $\alpha=90^{\circ}$ intervals. This was indicated in Figure 11 . High time resolution examples are given in Figure 13. Four pulses are indicated by vertical shading. The field variations have duration of 6 to $7 \mathrm{~s}$, comparable to the proton cyclotron frequency in the spacecraft frame. The pulses are essentially totally transverse oscillations (the bottom panel shows almost no variation in $|\mathrm{Bl}|)$. Analyses of a number of such events indicate that the waves typically propagate parallel to $\vec{B}, \theta_{\mathrm{kB}} \approx 5^{\circ}$ to $15^{\circ}$, and the waves are linearly or highly elliptically polarized. The transverse amplitudes are typically 1- to 3-nT (in an 8- to $12-\mathrm{nT}$ field), although peak-to-peak amplitudes as large as 5 to $7 \mathrm{nT}$ have been detected. At the present time, the generation mechanism of these unusual wave structures is unknown. It is uncertain whether they exist only for intervals where $\alpha \approx 80^{\circ}$ to $90^{\circ}$ or whether they are present during other conditions but are masked by the presence of large amplitude $\mathrm{H}_{2} \mathrm{O}$ group ion cyclotron waves. It is even unclear whether these structures are cometary in origin or not. The power associated with these isolated pulses is so small that standard techniques such as power spectral analyses cannot be used to determine their presence.

Although cometary power spectral analyses have strongly indicated peaks at the $\mathrm{H}_{2} \mathrm{O}$ group ion cyclotron frequency, both the ICE and Giotto magnetic field and plasma 


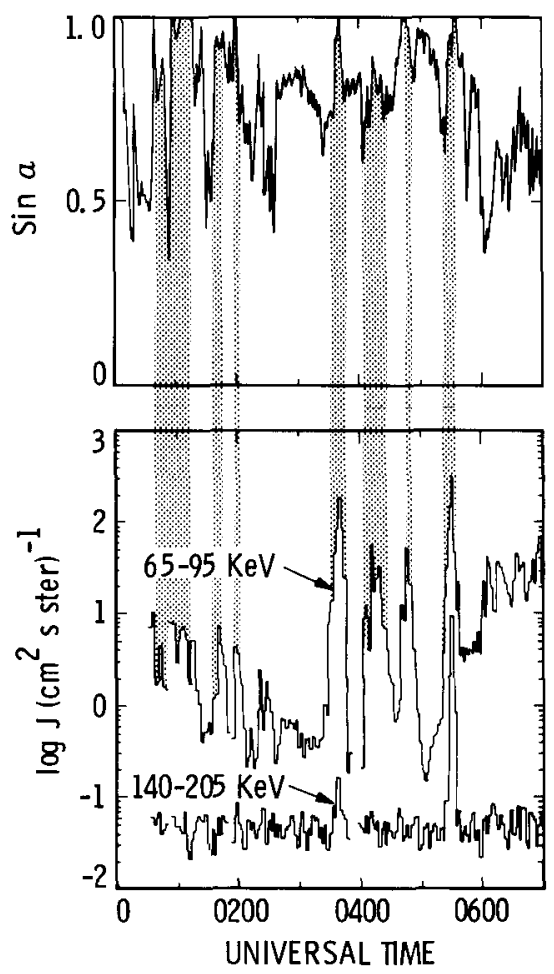

Figure 12. Sin $\alpha$ (the angle between $\vec{V}_{s w} \times \vec{B}$ ) and the logarithm of the energetic ion flux at Comet Giacobini-Zinner. When $\sin \alpha$ is large (shaded regions), the flux of the 65 - to $95-\mathrm{keV}$ ions is high. This is due to the pickup process discussed previously. Note that the ion flux is highly variable, indicating a general lack of pitch angle isotropy. From Tsurutani et al. (1989a).

observations have not found any waves (other than the above) associated with the pickup of cometary protons. Gary et al. (1988) have simulated the proton pickup process and have found that there is rapid isotropization of the protons into a stable "shell" distribution. The waves saturate at small amplitudes, explaining the lack of detectable waves. The heavy ions do not show similar effects because of the much larger mass of the ions.

2.2.5. The Nonresonant Ion Beam Instability. To date, there has been no confirmation of the onset of this instability at comets, although it could be expected under certain plasma conditions. The waves generated would have $\lambda \gg \lambda_{\text {res. }}$ Thus they would be hard to detect, in general. Long time intervals must be studied, and this necessarily involves averaging over different plasma spatial regions, perhaps leading to ambiguous results. It should be mentioned that in the stochastic acceleration theory of Galeev et al. (1990), such long wavelength waves are necessary to accelerate ions to the observed high energies. 

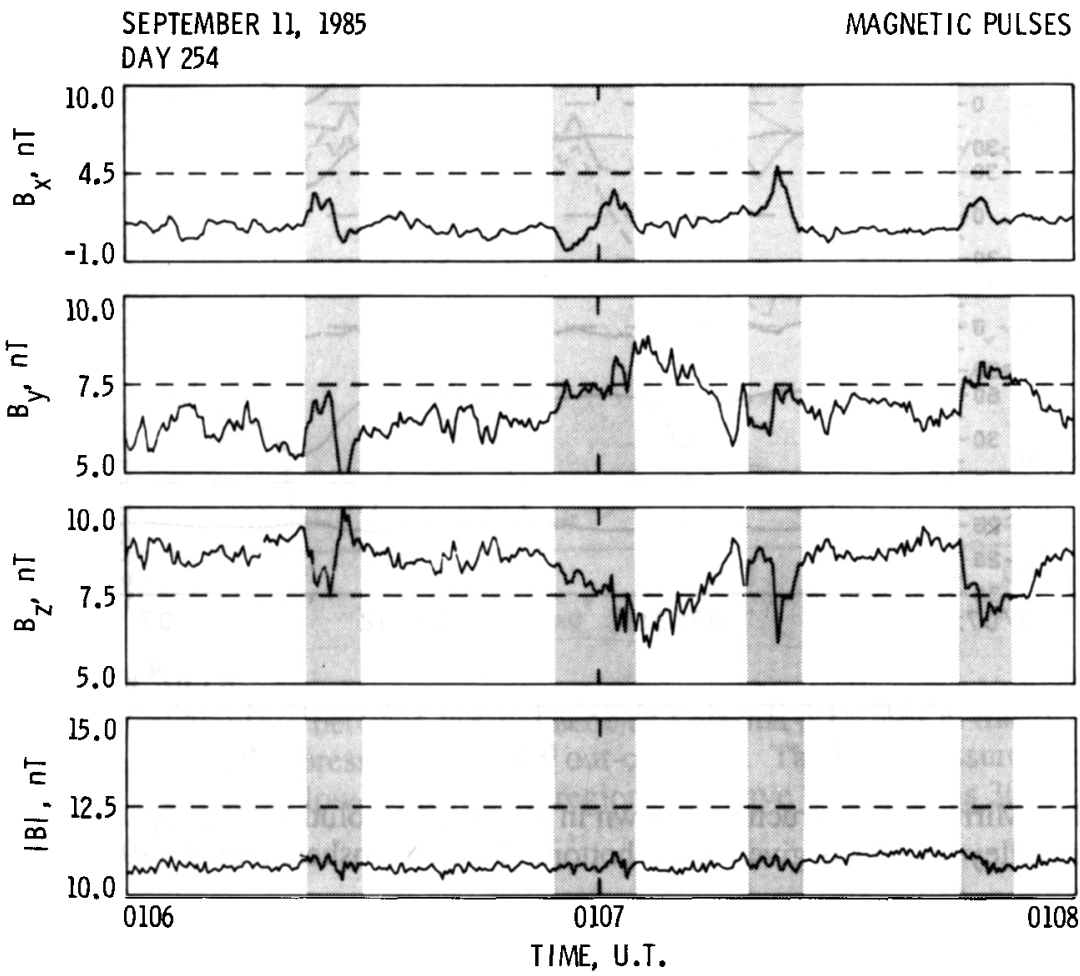

Figure 13. An example of transverse pulses in the magnetic field when $\alpha \approx 80^{\circ}$ to $90^{\circ}$ (shown in shading). The pulses have durations of 6 to $7 \mathrm{~s}$, comparable to the proton cyclotron period in the spacecraft frame. The pulses are typically linearly or highly elliptically polarized and are propagating at angles close to $\vec{B}$. The coordinate system is GSE. From Tsurutani et al. (1989b).

2.2.6. Drift Mirror Mode. Closer to the comet, within the magnetosheath, mirror mode waves have been detected by the VEGA magnetic field experiment. Figure 14 is from Yeroshenko et al. (1987). These observations were taken at a distance of $3 \times 10^{4}$ to $18 \times 10^{4} \mathrm{~km}$ from the comet, deep in the sheath region. These structures are characterized by irregular (nonsinusoidal) dips in the magnetic field.

Similar structures have also been detected at Giacobini-Zinner (Smith et al., 1987; Slavin et al., 1987) and were observed by Giotto as well (Glassmeier, private communication, 1989). However, the magnetic structures observed by ICE and Giotto were not nearly as striking as those found in the VEGA data set.

Price (1989) has demonstrated that the pickup ion ring distributions can drive this mode unstable. One major difference between the cometary ring case and that of the magnetosheath is that for the cometary case, maximum growth is predicted to occur at relatively small angles relative to $\vec{B}$. 


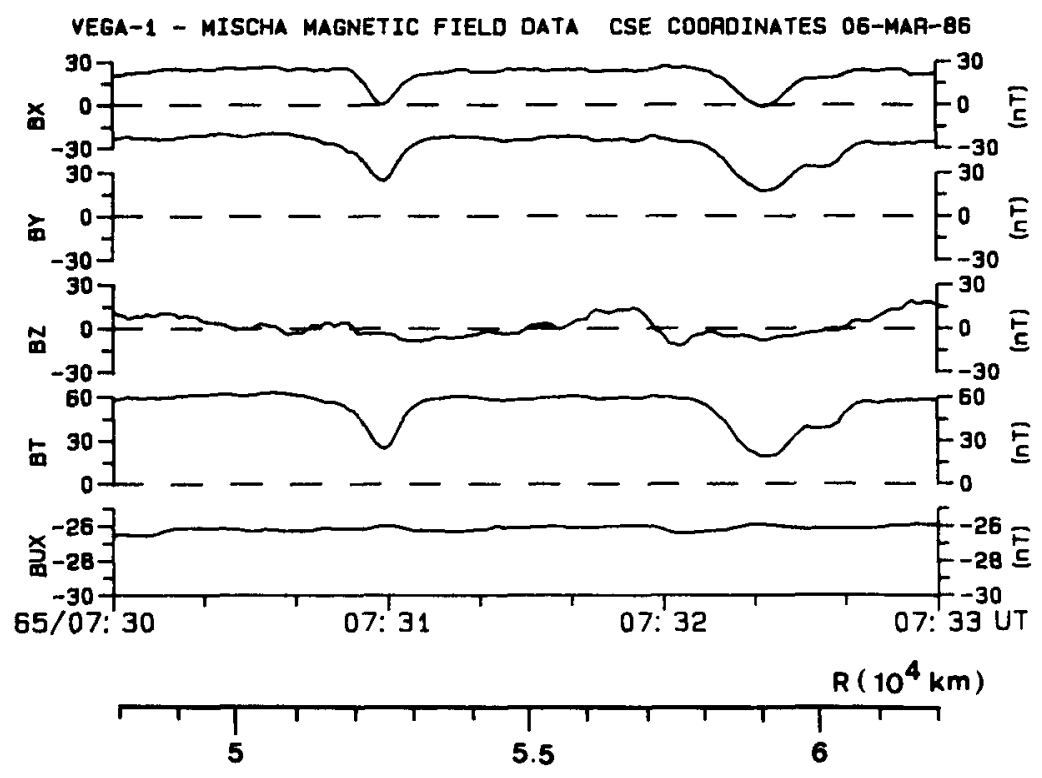

Figure 14. Mirror mode structures shown in high time resolution. The spacecraft distance from the Halley nucleus is given at the bottom. From Yeroshenko et al. (1987).

Russell et al. (1987) have speculated that these structures may be the cometary rays observed from ground-based telescopes, that extend to tens of millions of kilometers downstream of cometary comas. However, more theoretical effort is needed in this area. It remains to be shown that the observed brightness variations and the $\sim 10^{7}$ to $10^{8} \mathrm{~km}$ "wavelengths" can be generated from this mechanism. If the field-aligned wavelengths are considerably shorter, one would expect more of a "patchwork" pattern rather than elongated "tubes."

Mirror modes are one of the dominant structures in planetary magnetosheaths as well. They have been detected in the terrestrial, Jovian, and Saturnian magnetosheaths (Tsurutani et al., 1982).

Figure 15 shows the relationship between the magnetic and plasma pressure in mirror mode structures in the Earth's magnetosheath. These measurements were made by the ISEE-1 plasma and magnetic field instruments. The magnetic and plasma pressure peaks are $180^{\circ}$ out-of-phase, such that the total pressure is essentially constant. This anticorrelation causes the plasma $\beta$ to vary from values of 1 or 2 to 30 across these structures. Tsurutani et al. (1982) demonstrated that these structures are propagating at $60^{\circ}$ to $80^{\circ}$ relative to $\vec{B}$ and have scale sizes of $\sim 20$ to 50 proton gyroradii in the plasma frame. The scale sizes are somewhat larger than what is theoretically predicted (Hasegawa, 1975). This remains an important theoretical issue. 


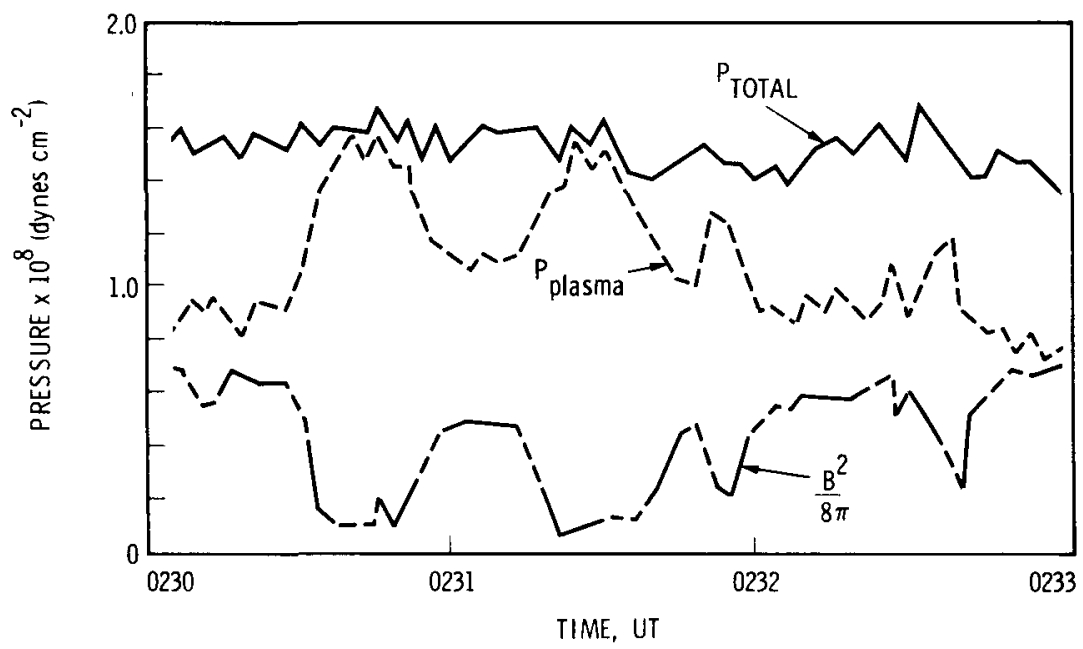

Figure 15. The relationship between the magnetic and plasma pressures in mirror mode structures. The two partial pressures are $180^{\circ}$ out-of-phase. The total pressure is constant. Because of this feature, the low-field, high- $\beta$ regions can have $\beta$ as large as 30 . From Tsurutani et al. (1982).

\section{Nonlinear Properties of the Low-Frequency Cometary Waves}

Figure 16 gives examples of the cometary waves near the bow shock of GiacobiniZinner. These waves have been detected from about twice the distance upstream of the bow shock to about half the distance to the nucleus. Specific features to note are: 1) the large amplitude, 100 -s "sawtooth" structures in the $\mathrm{B}_{\mathrm{Z}}$ component, 2 ) the significant magnetic field compression (bottom panel), and 3) high-frequency whistlers trailing the magnetic compression (in time). As stated previously, the transverse $\mathrm{H}_{2} \mathrm{O}$ group ion cyclotron $(100$-s) peak-to-peak amplitudes can be as large as $\Delta \vec{B} / \mathrm{B} \sim 2.0$, the magnetic compression $\Delta|B| / B=0.5$ or even higher, and the peak-to-peak whistler amplitudes $\Delta \vec{B} / B=1.5$. Waves with the above properties were detected only at Giacobini-Zinner.

Figure 17 shows a comparison between the plasma density and magnetic field magnitude for the $\mathrm{H}_{2} \mathrm{O}$ group ion cyclotron waves. There is a clear correlation between $|B|^{2}$ and density $(N)$, establishing that this is indeed the magnetosonic mode. Thus the steepened waves in Figure 16, which have the sharp decreases on the trailing edge, are propagating towards the Sun, but are convected past the spacecraft by the higher solar wind velocity. The whistler packets that are at the leading edge of the magnetosonic waves are also anomalously Doppler shifted and appear left-hand polarized in the spacecraft frame.

A simplified schematic of how a sinusoidal wave could possibly steepen is given in Figure 18. This has been adapted from Cohen and Kulsrud (1975). Note that the steepened nonlinear wave (bottom panel) has a $180^{\circ}$ phase rotation at the leading edge and an elongated portion at the trailing end. The trailing end of the wave contains the remainder of the $180^{\circ}$ phase rotation. This simple schematic is intended to give only a general descrip- 

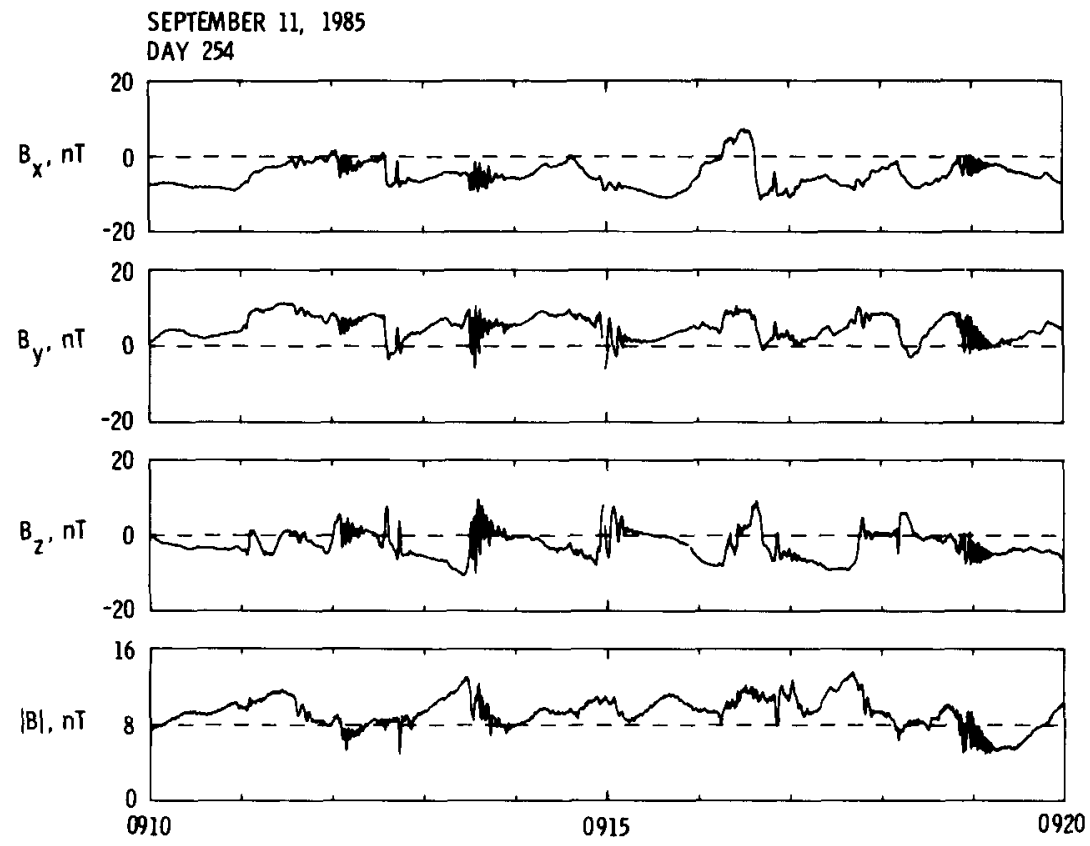

Figure 16. Cometary waves near the bow shock of Comet Giacobini-Zinner. The coordinate system is GSE. The 100 -s oscillations are the magnetosonic waves driven by the pickup of $\mathrm{H}_{2} \mathrm{O}$ group ions. The generating mechanism is the resonant ring-beam instability, and because the spacecraft is basically in the ion rest frame, the waves appear near the ion cyclotron frequency. The high-frequency $0.3-$ to $2.0-\mathrm{Hz}$ waves are whistler mode emissions. The magnetosonic waves have a peak-to-peak amplitude as large as 2.0 and a compressional component $\Delta|\mathrm{B}| / \mathrm{B} \approx 0.5$. The whistler mode waves occur at the steepened, leading edge of the magnetosonic waves. From Tsurutani et al. (1987b).

tion. Simulation results indicate that the distance between points 3 and 7 actually increases (N. Omidi, personal communication).

A high time resolution example of a steepened wave is given in Figures 19 and 20. Figure 19 shows the steepened wave in principal axis coordinates where the standard notation is used: $\mathrm{B}_{1}, \mathrm{~B}_{2}, \mathrm{~B}_{3}$ correspond to the field in the maximum, intermediate, and minimum variance directions, respectively. The steep "partial rotation" is observed from 1411:54 to 1412:04, indicated in the figure. The hodogram in Figure 20 shows that there is $\mathrm{a} \sim 180^{\circ}$ phase rotation associated with this phenomenon. The main component change is observed in $B_{1}$ from 1410:20 to 1411:26 U.T. It changes linearly with time and can be observed to cause a more or less linear variation in the hodogram.

An example of a fully developed upstream whistler is shown in Figure 21 in principal axis coordinates. The whistler begins at $\sim 0858: 42$ U.T. at about the peak magnitude 


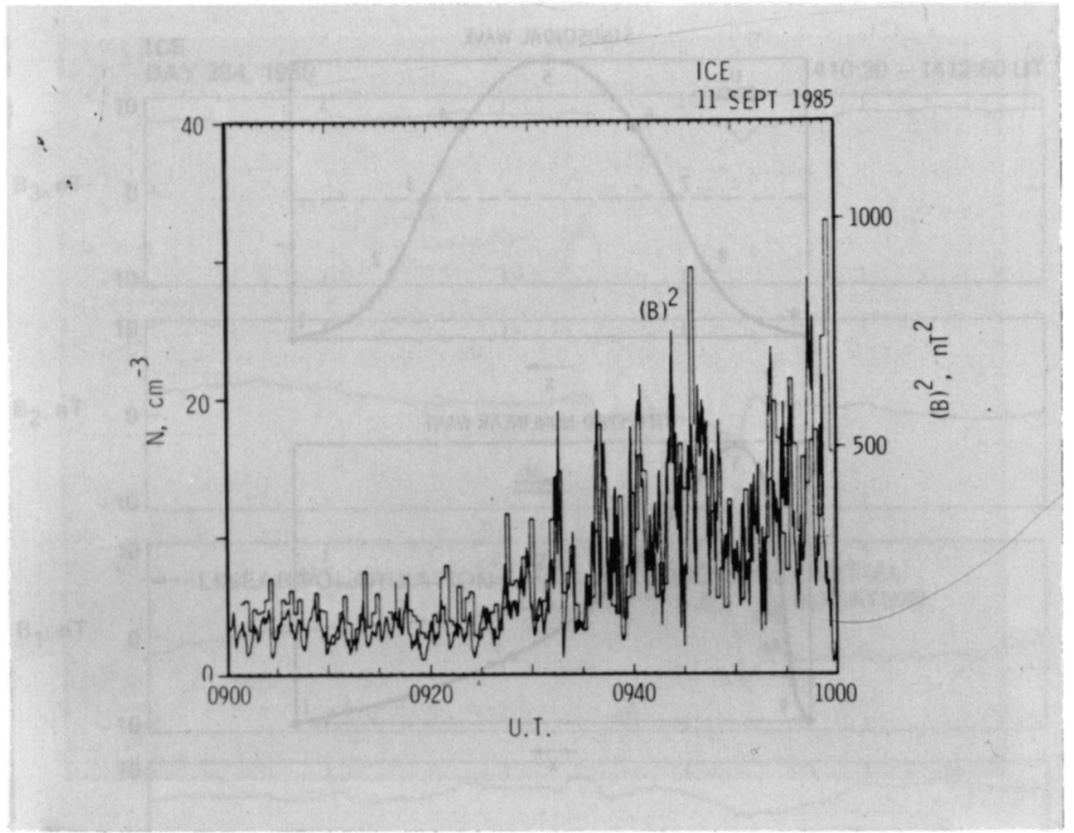

Figure 17. Relationship between the electron density and magnetic field (squared) at Comet Giacobini-Zinner. In this interval, these two parameters are well correlated, indicating that the fluctuations are magnetosonic in nature. Other intervals have less correlation. From Tsurutani et al. (1987b).

intensity of the compressional magnetosonic wave to which it is attached. The whistler decreases in amplitude linearly with time. The $\mathrm{B}_{1}$ component oscillates about a value of zero. Because the whistler is propagating close to parallel to the upstream ambient magnetic field direction $\left(\theta_{\mathrm{kB}}=10.9^{\circ}\right)$, the magnetic field magnitude decreases smoothly with time and distance. Two important points should be noted. First, it is apparent that the whistler is an integral part of the magnetosonic wave and it serves to decrease the field magnitude and alter the field direction. The partial rotation presumably has developed into a full wave packet. There is no longer an abrupt transition to the upstream ambient field, as occurs at a partial rotation. Second, the whistler acts to gradually reorient the steepened wave field to the ambient direction and also causes a broadening of the field magnitude gradient.

It should be noted that the evolution of a steepened magnetosonic wave to a wave with a "fully developed" upstream whistler packet discussed above is only a hypothesis based on wave "snapshots." There is no evidence of this sequence from spacecraft observations. A computer simulation would be quite useful for this purpose.

There have been several proposed sources for the whistlers. Perhaps the most clearly demonstrated mechanism is through the generation of dispersive whistlers, as suggested by Omidi and Winske $(1987 ; 1988 ; 1990)$ from hybrid simulation results. They have proposed a scenario for the time sequence of whistler development. An open question that still exists is the influence of other whistler generation mechanisms that have been proposed, such as those discussed by Goldstein and Wong (1987), Brinca and Tsurutani (1988b), and Kaya et al. (1989). The former two mechanisms involve generation by 

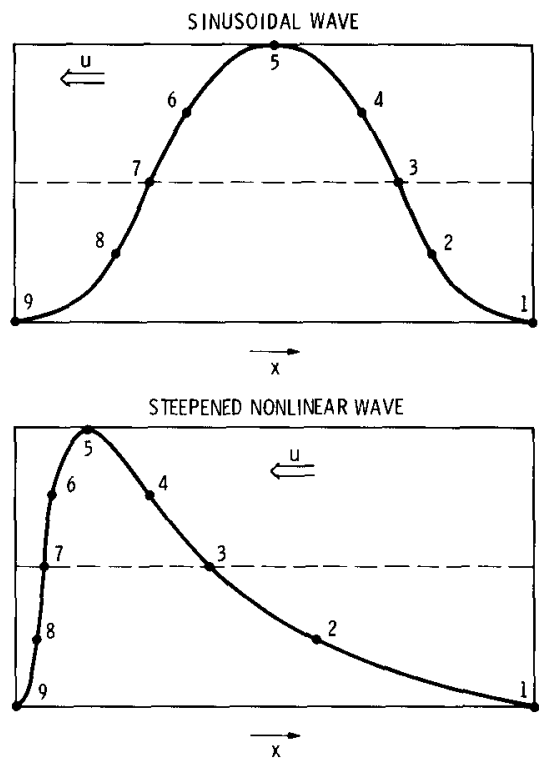

Figure 18. A schematic illustrating how a sinusoidal wave (top panel) could steepen to have a sharp phase rotation at its leading edge (bottom panel). From Tsurutani et al. (1987b). An adaptation from Cohen and Kulsrud (1975).

pickup of heavy ions and protons at the distorted steepened fronts of the magnetosonic waves, and the latter mechanism involves phase trapping of heavy ions by the whistler wave train. It would be important to establish the relative wave amplitude contribution for these various mechanisms. Preliminary simulation results in Omidi and Winske (1990) indicate that the generation of dispersive whistlers may account for the majority of the wave amplitude.

Even though the steepened magnetosonic waves appear to be complex (see above discussion), recent analyses indicate that in some cases, they are even more interesting. Figures 22 and 23 give an example of a steepened magnetosonic wave with regions of both phase rotation and lack of rotation. In the bottom panel of Figure 22, it can be noted that there is a slow (linear) magnetic magnitude increase throughout the wave form. However, the principal axis components have a different time (and distance) evolution. The components are constant from $0823: 38$ to 0824:44 U.T., or approximately one-half of the $\left(\mathrm{H}_{2} \mathrm{O}\right.$ group) wavelength (Figures 22 and 23). There is a small phase rotation in $\mathrm{B}_{2}$ from point 1 to 2 , but $B_{1}$ remains constant during this interval. $B_{1}$ has an abrupt rotation between points 3 and 4 and then remains constant until point 5 , where the "partial rotation" takes place. The rotation for $\mathrm{B}_{2}$ from 3 almost to 4 has an opposite sense, or a "back-rotation." This portion of the wave is right-hand polarized in the spacecraft frame, opposite to that of the whistlers. It does not seem possible that this shift is due to propagation effects. Such "reverse rotations" have been predicted to occur in solitons (Hada et al., 1989) and 


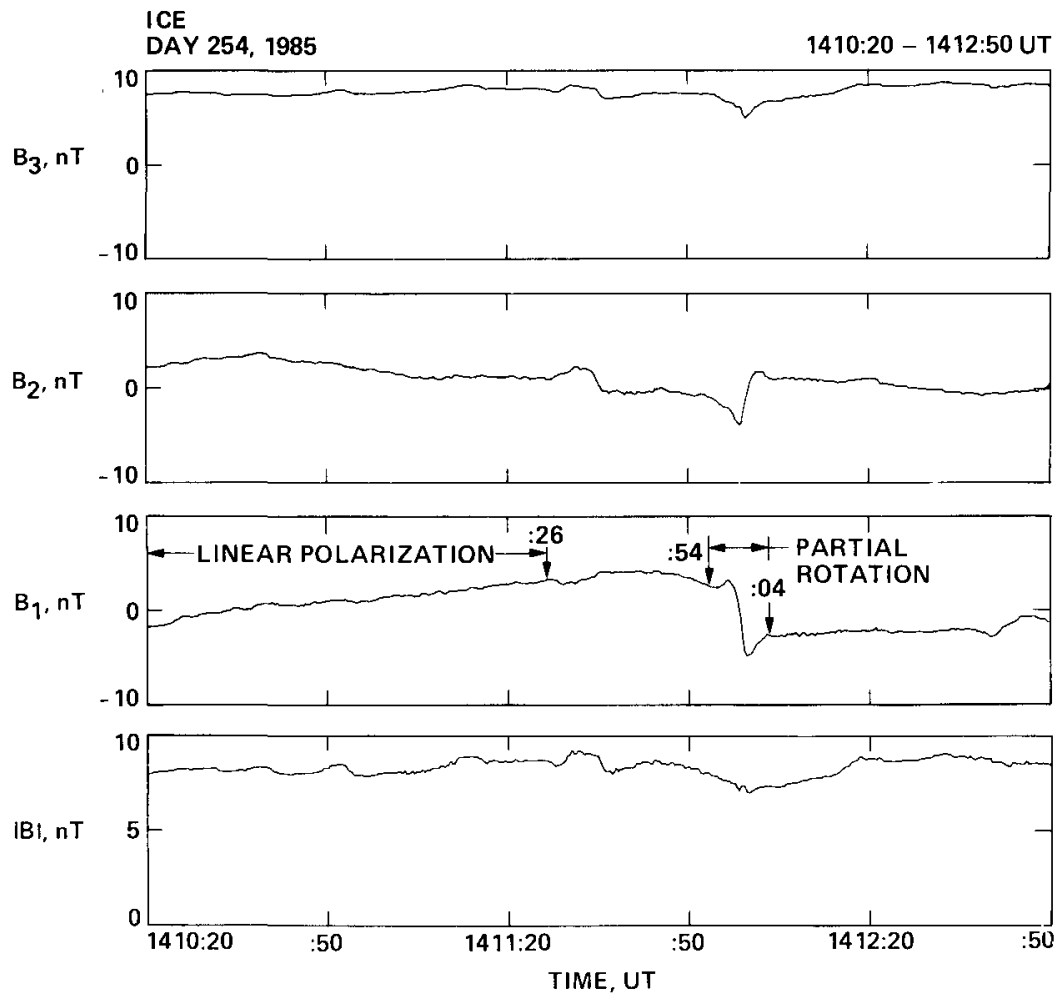

Figure 19. An example of a steepened magnetosonic wave. The steepened wave front is located at 1411:54 to 1412:04 U.T. This is a rotational discontinuity, and because it does not rotate a full $360^{\circ}$ in phase, it has been called a "partial rotation." The trailing portion of the wave, from 1410:20 to 1411:26 U.T., also contains some phase rotation, but because this is often small, this portion of the wave appears "linear" in polarization. The next figure, Figure 20, illustrates that all of the above features are part of the same wave. From Tsurutani et al. (1989c).

may have been detected in numerical simulations as well (Matsumoto, personal communication, 1989; Omidi, personal communication, 1989). The exact role this plays in the wave is not understood at this time.

The whistler packets are also not always as simple as the previous example shown. Figure 24 illustrates a whistler packet that is not propagating parallel to the magnetic field and therefore has a large magnetic compressional component associated with it. The $\vec{k}$ of individual cycles of the whistler waves were analyzed and compared with the direction of the upstream ambient magnetic field. It is found that the whistlers are propagating at $45^{\circ}$ to $51^{\circ}$ relative to the ambient field. It has been noted that in many cases, the direction of prop- 
ICE

DAY 254, 1985

$1410: 20-1412: 50$ UT
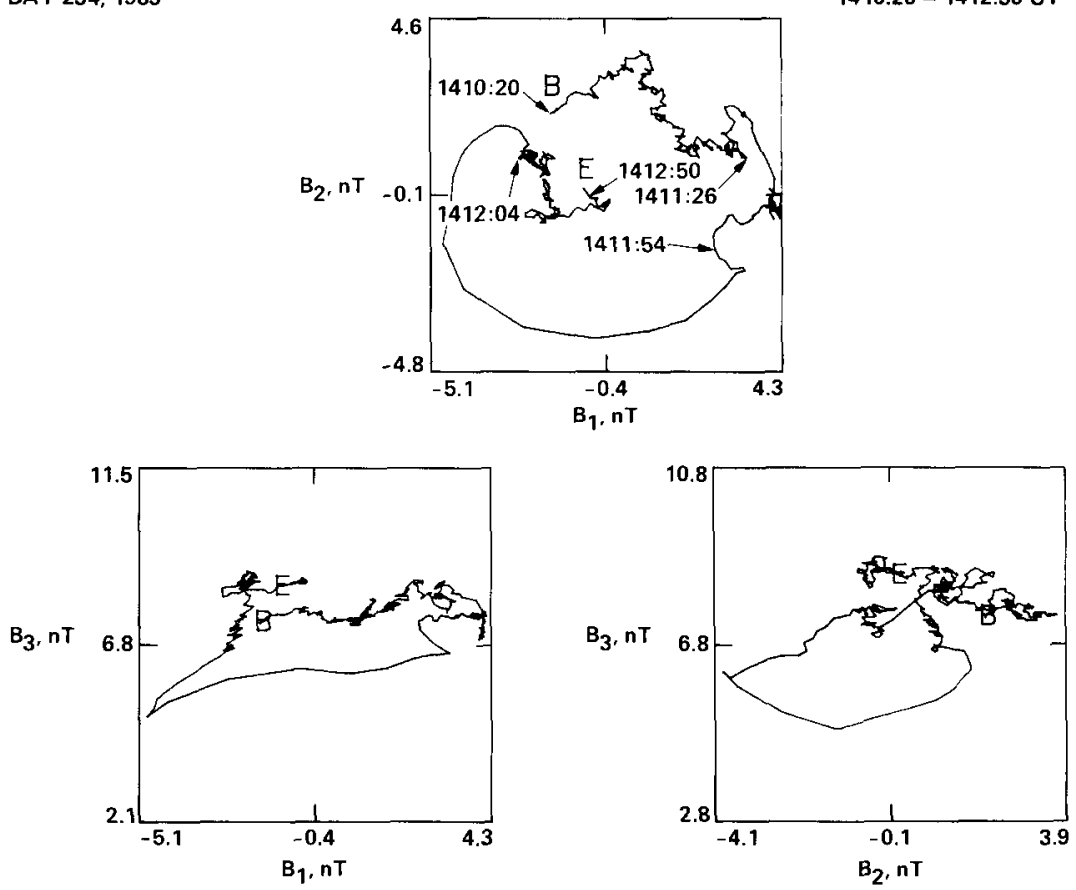

Figure 20. The hodograms for the steepened magnetosonic wave shown in Figure 19. $\mathrm{B}_{1}, \mathrm{~B}_{2}$ and $\mathrm{B}_{3}$ correspond to the field components in the maximum, intermediate, and minimum variance directions. Specific times are indicated in the $B_{1}-B_{2}$ plot so that the partial rotation and "linear" parts of the wave can be identified. The interval 1411:26 to 1411:54 U.T. contains significant phase rotation and may be an important feature of nonlinear steepened waves. Note that the total phase rotation is $360^{\circ}$. From Tsurutani et al. (1989c).

agation of the whistlers is not the same as that for the magnetosonic waves (Tsurutani et al., 1989c).

\subsection{COMPARISON TO THE EARTH'S FORESHOCK WAVES}

Nonlinear waves that are quite similar to those at comets have been detected in the Earth's foreshock (Hoppe et al., 1981). The generation mechanism for the long-period waves ( $20 \mathrm{~s}$ in the spacecraft frame) is established as being due to solar wind ions reflected at the bow shock, i.e., the right-hand resonant ring-beam instability.

The characteristics of the upstream waves are not as well known as the cometary waves at this time. It is believed that for the foreshock waves, simple, nonsteepened waves are observed close to the shock, and more complex cases (steepened with whistlers) 

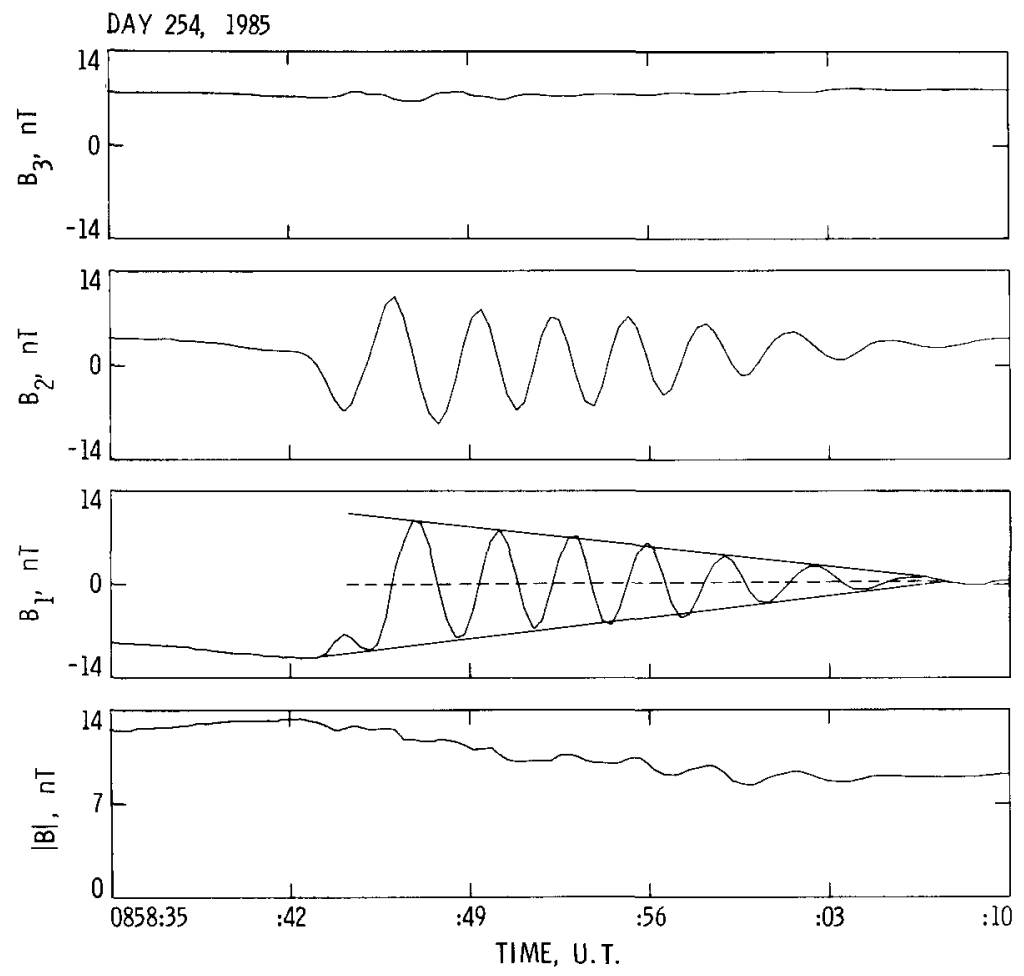

Figure 21. An example of an upstream whistler in principal axis coordinates. The whistler begins at the steepened edge of the magnetosonic wave and decreases linearly in amplitude with upstream distance. The whistler is present throughout the magnetic field magnitude gradient and presumably serves to broaden the gradient and reorient the magnetic field to the upstream ambient. From Tsurutani et al. (1989c).

are found further from the shock. Also, intrinsic "complex" left-hand polarized waves have been observed. The percent time this occurs and the nature of these waves are not certain at this time. It is unclear whether the left-hand waves are generated by the nonresonant instability, by some other mechanism such as three-wave processes, or from the formation of solitons, as discussed previously.

Spangler et al. (1988) and Leckband and Spangler (1989) have attempted to determine the cause of density fluctuations in the deep foreshock region. In addition to oblique wave propagation and magnetosonic wave steepening, they demonstrated that ponderomotive effects due to spatial gradients in the wave energy density are important. Leckband and Spangler (1989) have tentatively identified ion acoustic waves associated with the decay instability. 

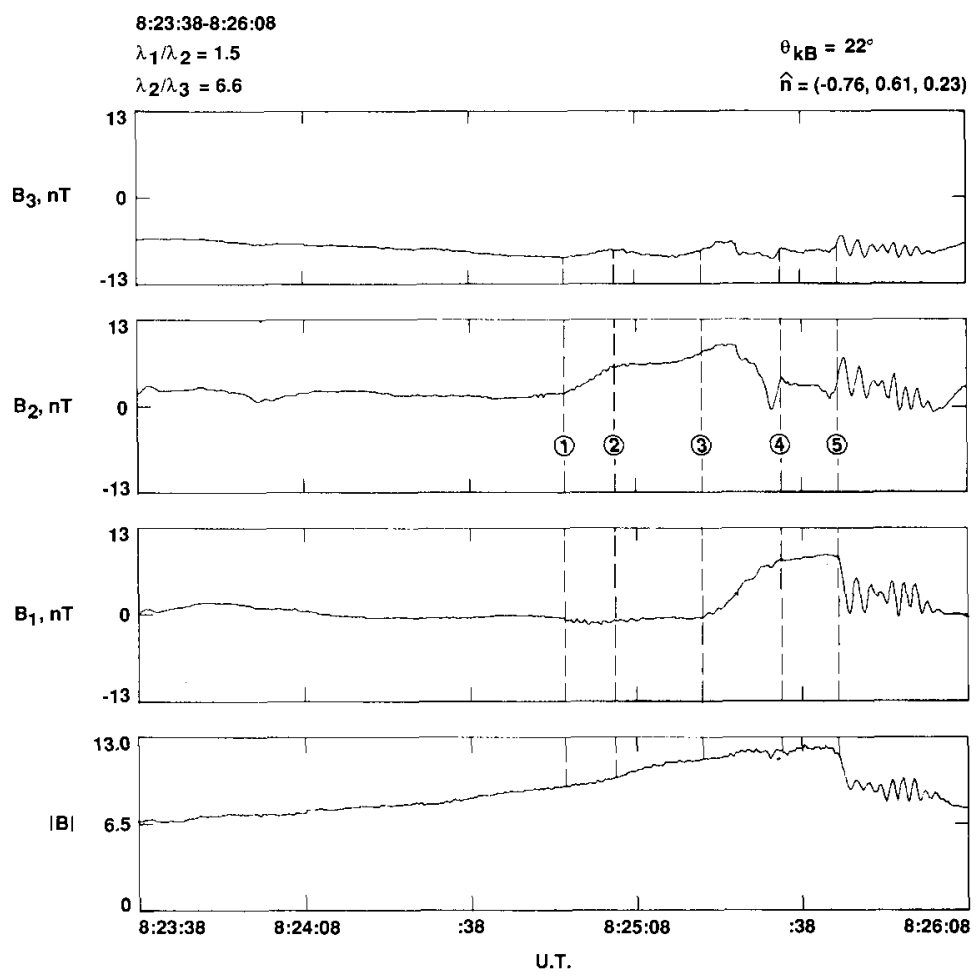

Figure 22. A more complex steepened magnetosonic wave case shown in principal axis coordinates. There are several distinct regions of wave phase changes, indicated in the figure. One region contains a "back rotation" where the wave polarization reverses for a short interval. This is shown in more detail in the hodogram of Figure 23. Such back rotations are not uncommon in the data set. Note that in the trailing portion of the wave, from 0823:38 to 0824:55 U.T., there is a gradual increase in B magnitude. However, there is essentially no change in wave phase $\left(\mathrm{B}_{1}\right.$ or $\left.\mathrm{B}_{2}\right)$. From Tsurutani and Smith (1989).

\subsection{WAVE CASCADING AND TURBULENCE?}

A high-frequency spectrum of wave power at frequencies above the $\mathrm{H}_{2} \mathrm{O}$ group ion cyclotron frequency is given in Figure 25. This spectrum was calculated when the angle between the IMF and $\mathrm{V}_{\mathrm{sw}}$ was close to $90^{\circ}$. $\mathrm{A} \mathrm{f}^{-2}$ line has been added to the figure. This spectral shape is significantly different than a Kolmogorov $f^{-5 / 3}$, or turbulence, spectrum (Glassmeier et al., 1989). Note that there is no apparent enhancement at the $\mathrm{H}_{2} \mathrm{O}$ group ion cyclotron frequency, $4 \mathrm{mHz}$, in this case. This is in sharp contrast to Figures 4 and 6 , where there is enhanced power at $\sim 10^{-2} \mathrm{~Hz}$. This lack of a "pump" wave is in agreement with the previous discussion of the lack of generation of (left-hand) ion cyclotron waves at large $\alpha$. It is also noted that the wave power is considerably below that in Figure 6 . 


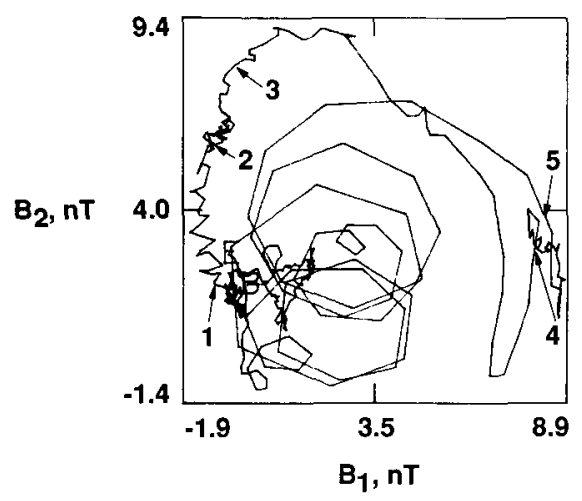

Figure 23. The $B_{1}-B_{2}$ hodogram for Figure 22. Note the intervals of discrete phase changes and the "back rotation."

Although it has been speculated by many authors that this power law dependence (varying from $5 / 3$ to 2 ) may be evidence for a cascade process, there are many other possible explanations that do not require such exotic (and unfortunately interesting) physical processes. Some of these are: 1) Although the spacecraft is nearly in the frame of the cometary ions, the spacecraft-comet relative velocity will cause small Doppler shifts in the waves. For ICE, the relative velocity was $21 \mathrm{~km} / \mathrm{s}$, and for the spacecraft armada to Halley, it was 68 to $79 \mathrm{~km} / \mathrm{s}$. 2) Glassmeier et al. (1989), Brinca and Tsurutani (1989), and Goldstein et al. (1990) have discussed the presence of cyclotron harmonic emissions. These modes would add power at the higher frequencies. 3) If the ions are not cold and have finite temperatures, this will shift the wave frequencies to higher values (Brinca and Tsurutani, 1988b). 4) Finally, it should be stressed that these waves are highly nonlinear. In the case of Giacobini-Zinner, they are steepened (and also have formed whistler precursors) such that higher and lower frequency power is generated. It is possible that some or all of the above processes could combine and give a relatively smooth power law spectrum.

Recent numerical simulations by Kojima et al. (1989) have led to further doubt that a cascade process is operative. These authors have demonstrated that strong wave-particle interactions may inhibit the decay instability and that wave-particle interactions may dominate the $\mathrm{H}_{2} \mathrm{O}$ group ion cyclotron wave evolution. Shapiro and Shevchenko (1988), have demonstrated that resonant wave-particle interactions and ion shell formation will lead to a wave power law spectrum with an index of $\sim 2.0$, similar to that of the observations. This may be a simple explanation for the observed spectra.

\subsection{WAVE PARTICLE INTERACTIONS}

The above nonlinear wave features open up a whole host of theoretical challenges concerning the interaction of these low-frequency waves with the solar wind plasma or with energetic particles. Current models assume parallel propagating, noncompressive 

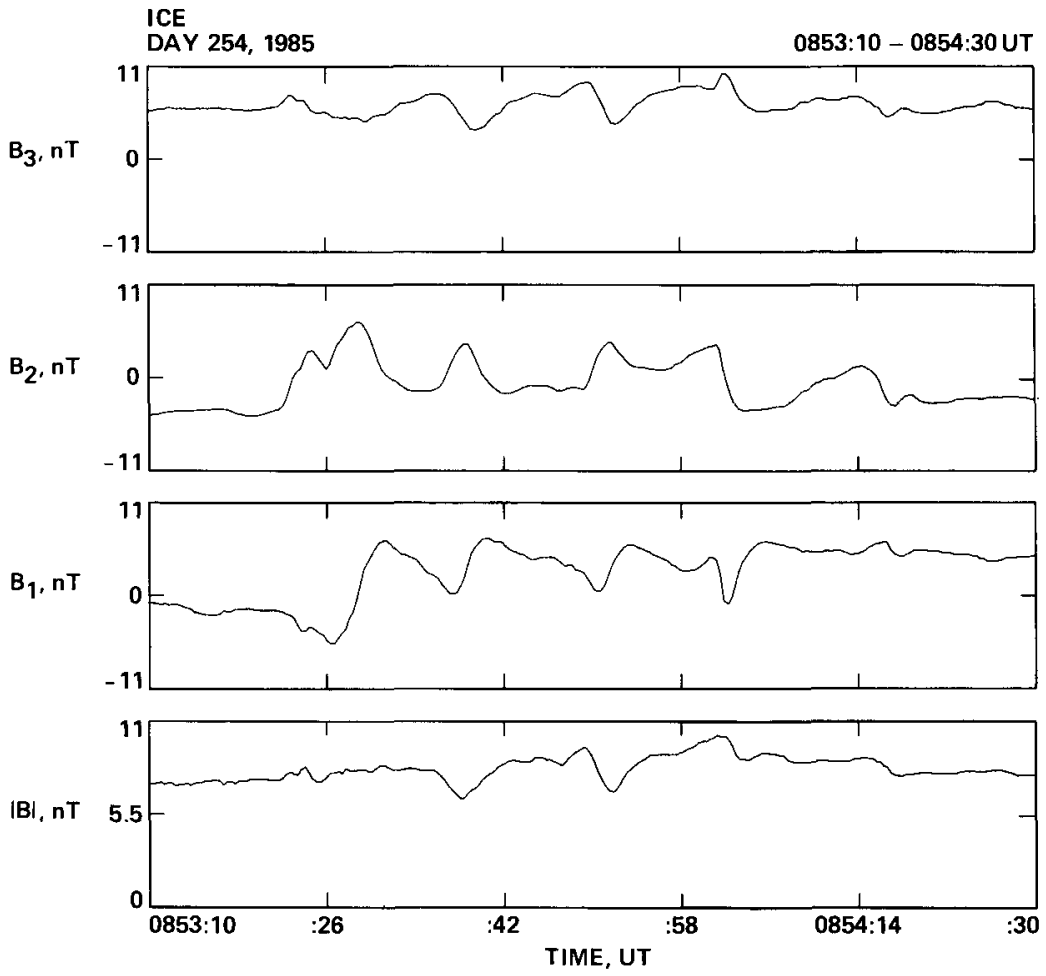

Figure 24. An example of a complex whistler packet. Not all whistlers propagate relatively parallel to $\vec{B}$. This is one such example. These whistlers are propagating at $45^{\circ}$ to $51^{\circ}$ relative to $\vec{B}$. Note the large compressional component associated with the waves.

waves travelling at the Alfvén speed (Terasawa, 1989). None of these assumptions are consistent with the data.

The wave amplitudes are highly nonlinear, $\Delta \vec{B} / \mathrm{B} \approx 1.0-2.0$, a feature that is not accommodated by present wave-particle interaction theories. The waves have significant compressive components, a feature that should lead to enhanced particle mirroring. If the waves propagate at sufficiently large angles to the ambient magnetic field, there could be gradient B drift acceleration, particularly for energetic protons that have relatively small gyroradii (see the general discussion and references in Armstrong et al., 1985). The whistler mode waves and the "partial rotations" are small on the scale of the water group ion gyroradius. The interaction of energetic particles with such structures would cause a

(nonresonant) breaking of the first adiabatic invariant, a topic not studied in much depth to date. The nonlinear steepening of the magnetosonic waves leads to the creation of higher frequency waves (previously discussed) and also longer wavelength emissions. The latter are particularly important for the acceleration of the high-energy tail of the ion distribution. Finally, I mention that steepened magnetosonic waves are propagating at speeds much 


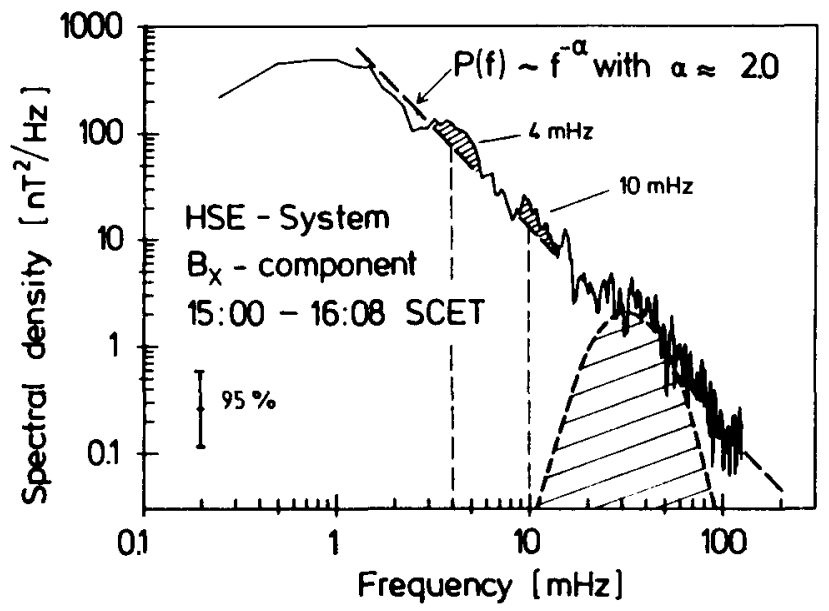

Figure 25. A power spectrum of a transverse component of the magnetic field fluctuations at Comet Halley. The coordinate system is the same as that described in Figure 6. The interval analyzed occurred when $\vec{B}$ was nearly orthogonal to the solar wind velocity. The spectrum can be fit with a $\mathrm{f}^{-2.0}$ power law, indicating that it does not fit the KolmogorovObukov turbulence prediction. There is no obvious peak at the $\mathrm{H}_{2} \mathrm{O}$ group ion cyclotron period $(4 \mathrm{mHz})$, in agreement with the results in Figure 11 for Comet Giacobini-Zinner. The wave power is lower by an order of magnitude than that shown in Figure 6. From Glassmeier et al. (1989).

larger than the Alfvén speed. Although, small-amplitude magnetosonic waves propagate at the MHD fast speed, the speed of the steepened waves may be two to three times the Alfvén speed. It is best to use the results of numerical simulations to get good values for this velocity.

According to the simulations of Winske and Gary (1986), it has been estimated that $30 \%$ to $50 \%$ of the particle pickup energy goes into waves. An estimate of the wave energy at Giacobini-Zinner is $5 \times 10^{15}$ joules (Tsurutani and Smith, 1986b) and at Halley, $1.8 \times 10^{17}$ joules. Relevant questions are: Does part of this energy go back into energizing the cometary pickup ions via energy diffusion through stochastic acceleration or via transit time damping? Are the waves damped via solar wind protons, leading to a hotter solar wind (Sharma et al., 1988; Hizanidis et al., 1988), or do the waves simply propagate away? A wave energy budget is needed to understand these potentially important waveparticle interaction effects.

All of the above features are relevant for wave-particle interactions, particularly for stochastic Fermi acceleration processes. Some of these features may be difficult to apply to an acceleration model, but these features represent plasma reality. It will first be necessary to examine these various wave phenomena to determine how important they are, e.g., what effect they have on the overall wave-particle interaction process of interest. The most important processes should be included into the appropriate models. An excellent start in this 
area is the recent work by Cravens (1989). It will also be particularly challenging to determine if the effects of these features can be put into analytic form.

\section{ELF and VLF Plasma Waves}

There were ELF (10 to $1500 \mathrm{~Hz})$ and VLF $\left(10^{3}\right.$ to $\left.10^{6} \mathrm{~Hz}\right)$ plasma wave detectors placed on four spacecraft flown to comets: ICE (Scarf et al., 1978), Sakigake (Oya et al., 1986), and the two VEGAs (Grard et al., 1986). The ICE electrostatic wave antennas were an order of magnitude larger than the others, leading to approximately an order of magnitude greater sensitivity. Only ICE had the capability of measuring the electrostatic noise associated with thermal electron motion. This was used by Meyer-Vernet et al. (1986) to obtain the plasma density in the cold ion tail of Giacobini-Zinner. Terrestrial kilometric radiation can also be measured by this instrument.

In addition to electric field measurements, both ICE and Sakigake had search coil sensors, which are used to measure ELF magnetic waves. Because the Sakigake coil was mounted on the spacecraft body, the spacecraft interference was too high to make useful measurements.

Figures 26 and 27 give the wave measurements from VEGA 2 and VEGA 1, respectively. In Figure 26, the solid curve is the average intensity, and the "line" is two standard deviations higher. The vertical scale is -70 to $10 \mathrm{~dB}$, where $0 \mathrm{~dB}$ is 1 volt rms. At 0720 U.T., there was probably a dust impact on the sensor. The measurements are not reliable beyond this time.

Figure 27 gives the VEGA wave spectrum for a variety of distances from the comet. The wave intensity increases with decreasing distance from the comet, consistent with the results in Figure 26. Curve a) corresponds to a distance of $1.8 \times 10^{5} \mathrm{~km}$, and

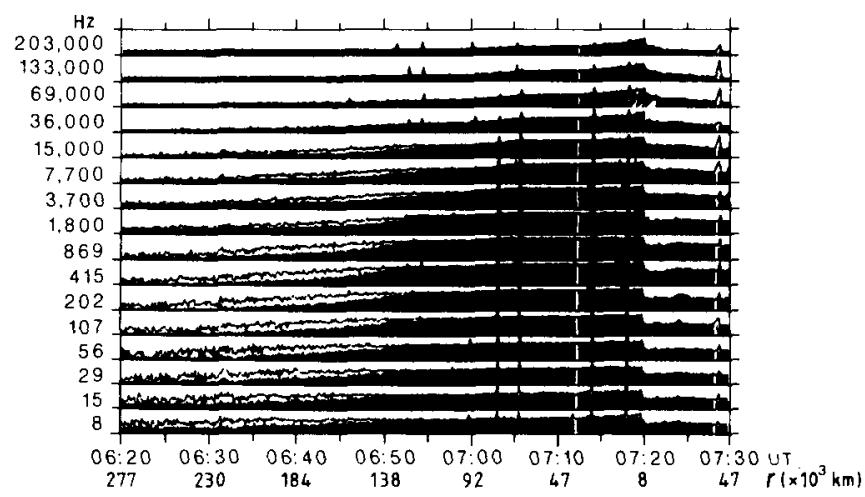

Figure 26. VEGA ELF/VLF wave measurements close to Comet Halley. The solid curve is the average intensity, and the line above, the $2 \sigma$ value for each 20 -s interval. The dynamic range for each channel is -70 to $10 \mathrm{~dB}$ with $0 \mathrm{~dB}$ corresponding to $1 \mathrm{~V}$ root mean square. The numbers at the very bottom are the distances from the nucleus. From Grard et al. (1986). 


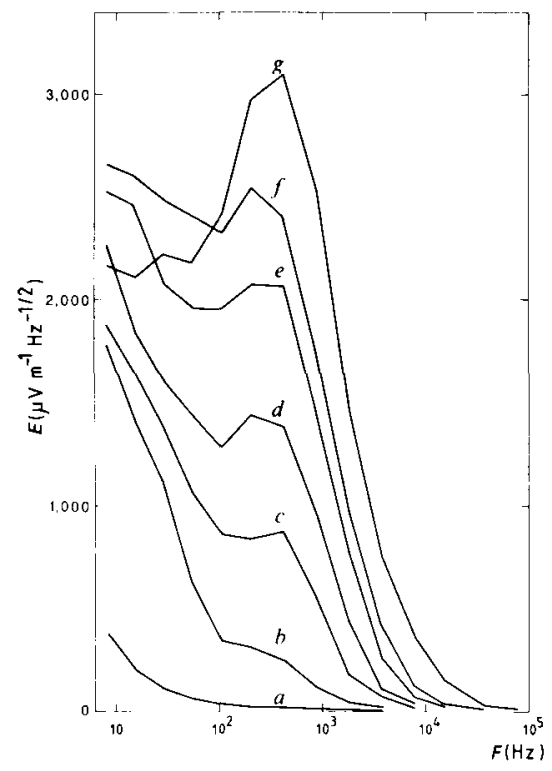

Figure 27. VEGA 1 ELF/VLF 2-minute averaged wave spectra close to Comet Halley. The various curves correspond to distances from the nucleus. Curves a) through g) correspond to $1.8 \times 10^{5}, 1.4 \times 10^{5}, 1.2 \times 10^{5}, 1.1 \times 10^{5}, 9 \times 10^{4}, 6 \times 10^{4}$, and $4 \times 10^{4} \mathrm{~km}$, respectively. A maximum in wave power is observed at $200 \mathrm{to} 400 \mathrm{~Hz}$. From Grard et al. (1986).

curve $\mathrm{g}), 4 \times 10^{4} \mathrm{~km}$. The waves have a maximum in a frequency range of 200 to $400 \mathrm{~Hz}$ for all spectra.

The overview of the Giacobini-Zinner cometary waves is shown in Figure 28, taken from Scarf et al. (1986b). Both the electric and magnetic wave intensities are given. It is obvious that waves generated by cometary pickup ions and photoelectrons are present for at least $10^{6} \mathrm{~km}$ and perhaps $2 \times 10^{6} \mathrm{~km}$ distance from the comet nucleus and more. Scarf et al. (1986b) stated that these emissions were the highest ever detected during the lifetime of the spacecraft, including solar flare intervals, at interplanetary shocks, and within the Earth's geomagnetic tail.

A comparison of the ICE Giacobini-Zinner and the Sakigake Halley wave intensities is given in Figure 29. Although Sakigake was at greater distances, $8 \times 10^{6} \mathrm{~km}$, the wave intensities were an order of magnitude higher.

Kennel et al. (1986) compared the plasma waves near Comet Giacobini-Zinner to those at interplanetary shocks and at the Earth's bow shock. The ELF/VLF wave signatures are almost completely analogous. Figure 30 illustrates the cometary wave features at and near the inbound and outbound shocks. Upstream of the shocks are electron plasma oscillations and ion acoustic waves, and there is broadband turbulence in the downstream regions. Similar features are found at all collisionless shocks detected in space. 


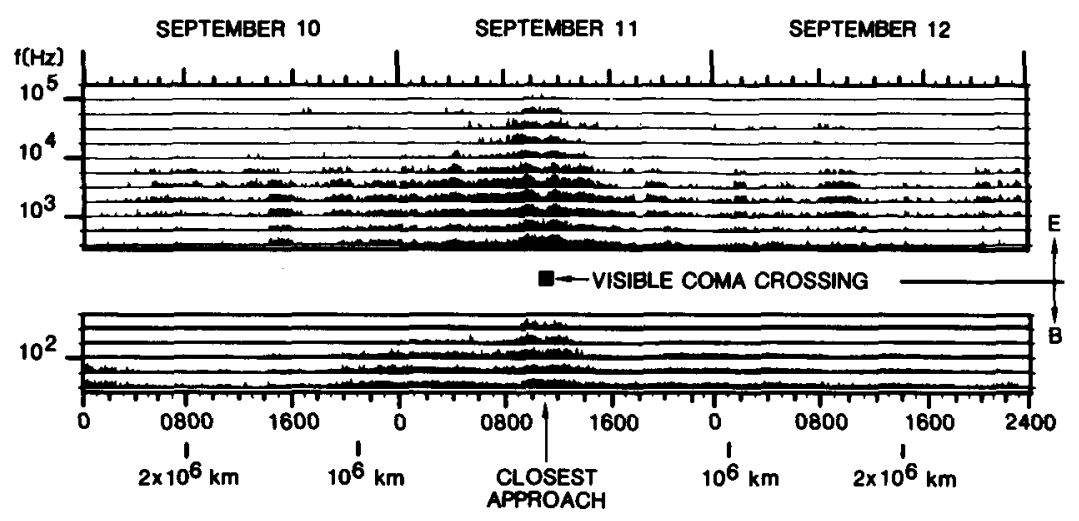

Figure 28. An overview of the electric and magnetic components of the Giacobini-Zinner ELF/VLF waves. Peak wave intensities are located close to the visible coma crossing. Cometary waves extend to $1 \times 10^{6}$ to $2 \times 10^{6} \mathrm{~km}$ from the nucleus. These emissions were the highest ever detected during the lifetime of the 11-year ICE/ISEE-3 mission. From Scarf et al. (1986b).

These cometary wave signatures led Scarf et al. (1986b) to immediately identify the cometary structure as a bow shock, whereas the magnetic field and plasma signatures were far less obvious (Smith et al., 1986a; Bame et al., 1986). More thorough analyses (Jones et al. 1986; Tranquille et al., 1986; Smith et al., 1986b) have given additional support to the Scarf et al. (1986b) shock hypothesis. Recently, Omidi and Winske $(1988,1990)$ have interpreted this transition region as consisting of a series of weak shocks rather than a single shock structure. This important issue has not been resolved.

A summary figure of some of the wave modes at comets is given in Figure 31. The top two left-hand panels illustrate the presence of electron plasma oscillations (EPO) and ion acoustic emissions discussed in the previous figures. Electromagnetic whistler modes are observed below the electron cyclotron frequency (lower right-hand panel). At (and below) the low-frequency end of the range of the search coils sensor are lower hybrid resonance (LHR) waves (in the center, bottom panel). The LHR emissions are discussed in more detail in Coroniti et al. (1987).

Recently, Richardson et al. (1989b) found a correlation between the presence of magnetic fields directed parallel to the solar wind velocity and electrostatic bursts at frequencies between $300 \mathrm{~Hz}$ and $10.0 \mathrm{kHz}$. The parallel field orientations are caused by the distorted fields associated with the steepened magnetosonic waves. This is shown in Figure 32. The electrostatic bursts thus have a quasiperiod of $\sim 100 \mathrm{~s}$. Brinca et al. (1989) have explained the generation of the electrostatic emissions by the pickup of cold cometary photoelectrons. 


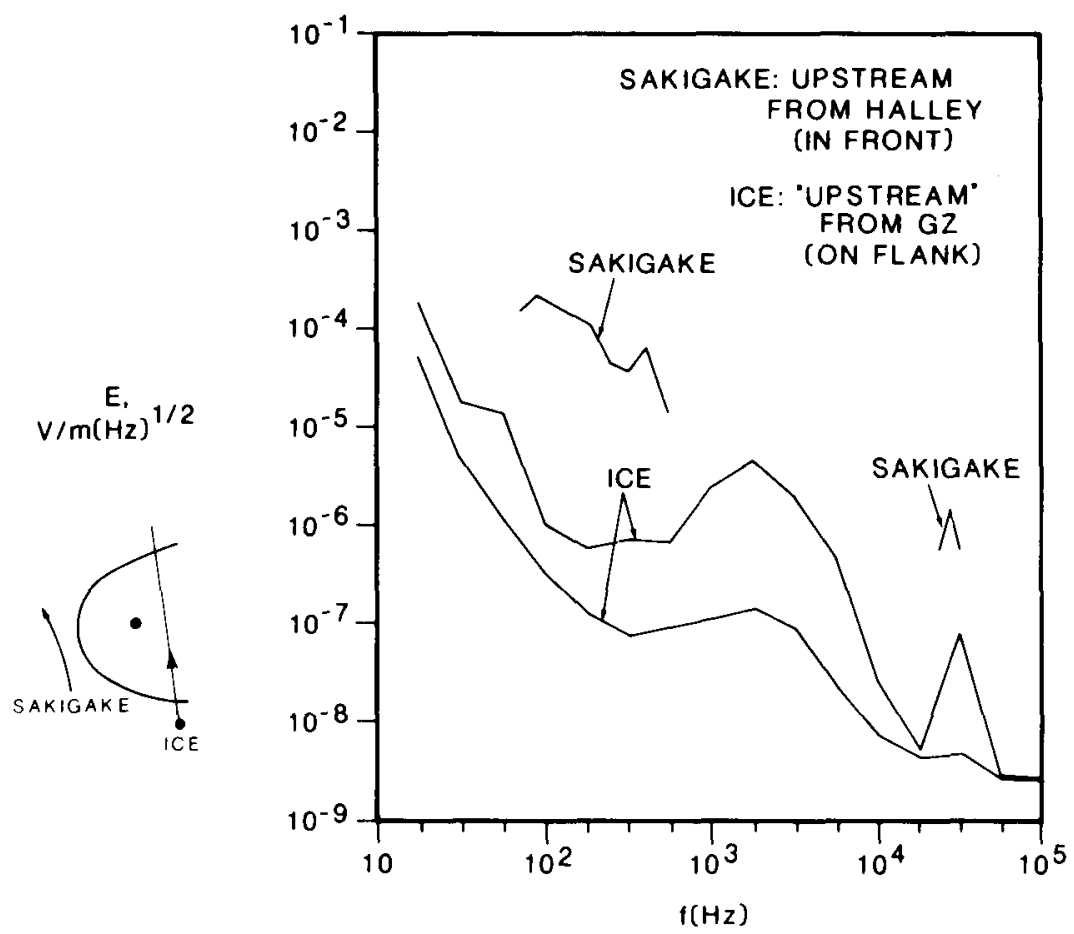

Figure 29. A comparison of the Sakigake (Halley) and ICE (Giacobini-Zinner) wave intensities. The Halley emissions were about one order of magnitude more intense. From Scarf (1989).

\section{Final Comments}

I have tried to give an up-to-date review and commentary on low-frequency, ELF, and VLF waves at comets. I have also emphasized currently unsolved problems. It is clear that although many of the basic wave modes and instabilities have been identified, there is still much more work to be done. Nonlinear wave phenomena and wave-particle interactions are exciting developing fields, stimulated by cometary research. Both further observational and theoretical efforts are necessary to understand possible implications. The next few years should be exciting ones for cometary plasma studies.

ACKNOWLEDGMENTS. Portions of this paper represent work done at the Jet Propulsion Laboratory, California Institute of Technology, under contract with the National Aeronautics and Space Administration. I wish to thank N. Omidi, S. P. Gary and the two referees for their helpful comments on this paper. 


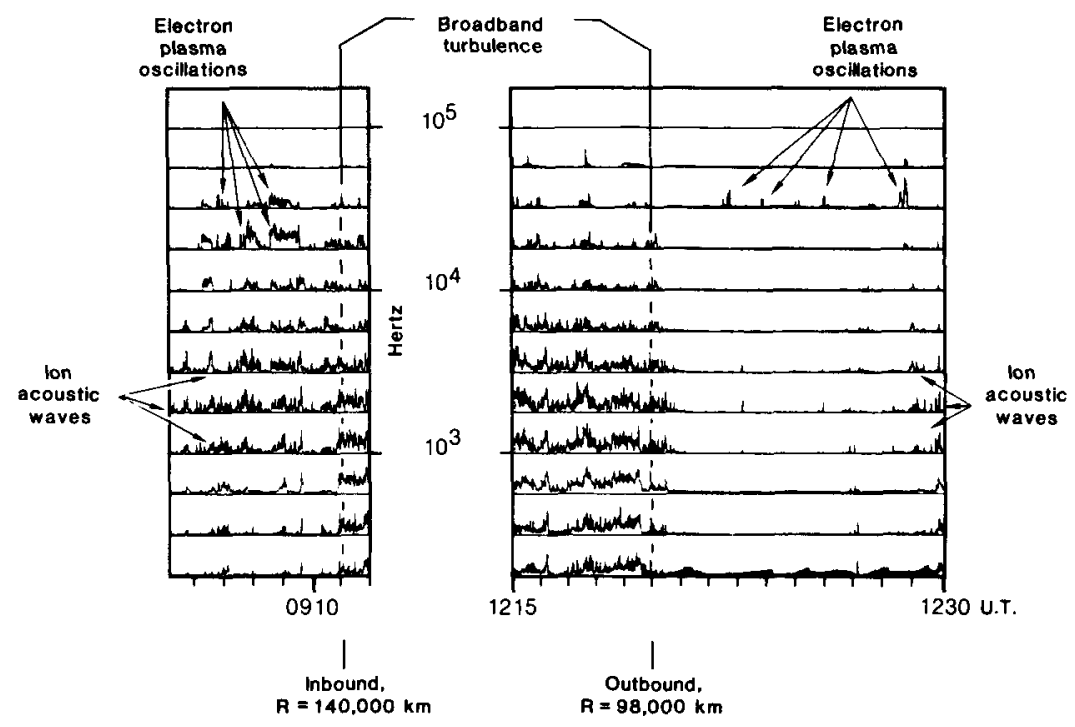

Figure 30. Plasma waves near the inbound and outbound crossing of the GiacobiniZinner bow shock. Upstream electron plasma oscillations and ion acoustic waves and downstream broad-band turbulence are observed at both crossings. These wave modes are typical of interplanetary shocks and the Earth's bow shock. Taken from Kennel et al. (1986).

\section{References}

Astron. and Astrophys. (1987) Comet Halley issue 187.

Armstrong, T.P., M.E. Pesses and R.B. Decker (1985) 'Shock Drift Acceleration,' in

Collisionless Shocks in the Heliosphere: Review of Current Research, ed. B.T.

Tsurutani and R.G. Stone, Am. Geophys. Un. Monograph 35, 271.

Balsiger, H., K. Altwegg, F. Buhler et al. (1986) 'Ion Composition and Dynamics of

Comet Halley,' Nature, 321, 330.

Bame, S.J., R.C. Anderson, J.R. Asbridge, D.N. Baker, W.C. Feldman, S.A. Fuselier,

J.T. Gosling, D.J. McComas, M.F. Thomsen, D.T. Young, and R.D. Zwickl (1986)

'Comet Giacobini-Zinner: Plasma Description,' Science, 232, 356.

Barnes, A. (1979) 'Hydromagnetic Wave and Turbulence in the Solar Wind' in Solar

System Plasma Physics, 1, ed. by E.N. Parker, C.F. Kennel and L.J. Lanzerotti, 251.

Brinca, A.L., and B.T. Tsurutani (1987a) 'On the Polarization, Compression, and Non-

Oscillatory Behavior of Hydromagnetic Waves Associated With Pickup Ions,'

Geophys. Res. Lett., 14, 495.

Brinca, A.L., and B.T. Tsurutani (1987b) 'Unusual Characteristics of Electromagnetic

Waves Excited by Cometary Newborn Ions With Large Perpendicular Energies,'

Astron. Astrophys., 187, 311. 


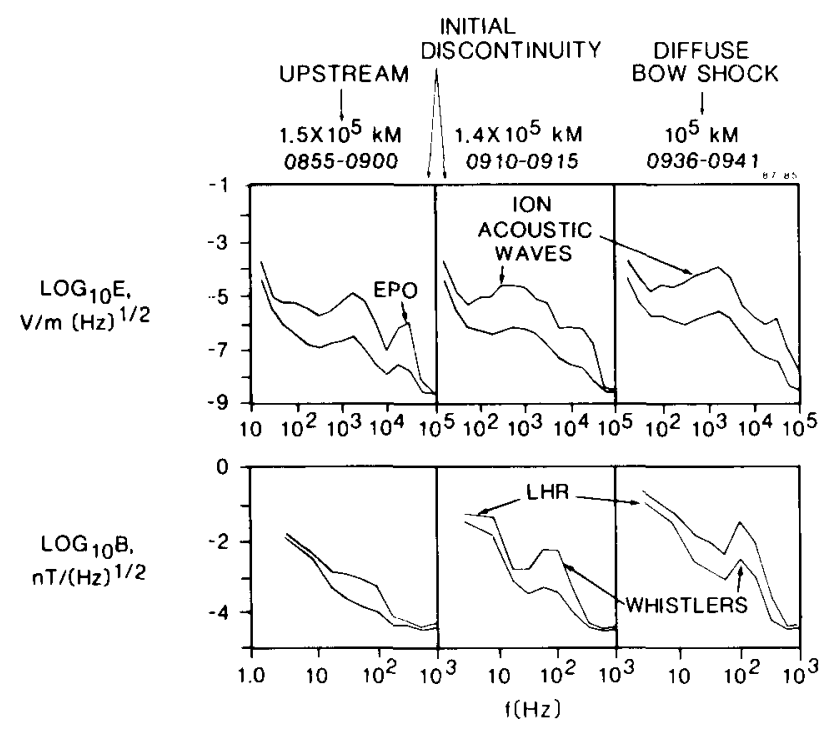

Figure 31. A summary of different ELF/VLF wave modes detected upstream of, at, and downstream of the Giacobini-Zinner bow shock. From Scarf (1989).

Brinca, A.L., and B.T. Tsurutani (1988a) 'Survey of Low-Frequency Electromagnetic Waves Stimulated by Two Existing Newborn Ion Species,' J. Geophys. Res., 93, 48. Brinca, A.L., and B.T. Tsurutani (1988b) 'Temperature Effects on the Pickup Process of Water-Group and Hydrogen Ions: Extension of "A Theory for Low-Frequency Waves Observed at Comet Giacobini-Zinner" by M.L. Goldstein and H.K. Wong, J. Geophys. Res., 93, 243.

Brinca, A.L., and B.T. Tsurutani (1989) 'On the Excitation of Cyclotron Harmonic Waves by Newborn Heavy Ions,' J. Geophys. Res., 94, 5467.

Brinca, A.L., B.T. Tsurutani, and F.L. Scarf (1989) 'Local Generation of Electrostatic Bursts at Comet Giacobini-Zinner: Modulation by Steepened Magnetosonic Waves,' J. Geophys. Res., 94, 60.

Chandrasekhar, S., A.N. Kaufman, and K.M. Watson (1958) 'The Stability of the Pinch,' Proc. Roy. Soc. A, 245, 435.

Cohen, R.H., and R.M. Kulrud (1975) 'Nonlinear Evolution of Parallel Propagating Hydromagnetic Waves,' Phys. Fluids, 17, 2215.

Coroniti, F.V., C.F. Kennel, F.L. Scarf, E.J. Smith, B.T. Tsurutani, S.J. Bame, M.F. Thomsen, R. Hynds, and K.P. Wenzel (1986) 'Plasma Wave Turbulence in the Strong Coupling Region at Comet Giacobini-Zinner,' Geophys. Res. Lett., 13, 869.

Cravens, T.E. (1989) 'Test Particle Calculations of Pick-Up lons in the Vicinity of Comet Giacobini-Zinner,' Planet. Space Sci., 37, 1169.

Crooker, N.U., and G.L. Siscoe (1977) 'A Mechanism for Pressure Anisotropy and Mirror Instability in the Dayside Magnetosheath,' J. Geophys. Res., 82, 185. 


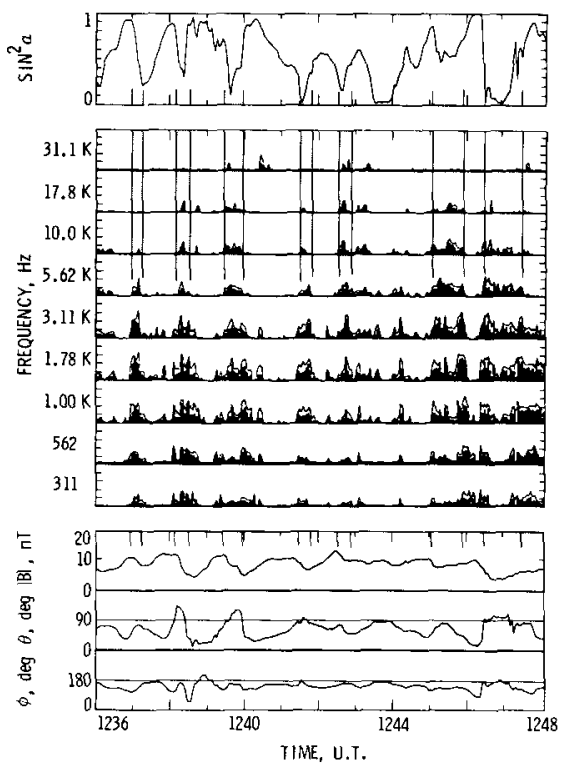

Figure 32. The relationship between bursts of ELF/VLF electrostatic bursts (middle panel) and $\sin ^{2} \alpha$ decreases (top panel) caused by steepened magnetosonic waves (bottom panel). The correlated intervals are denoted by shading. From Richardson et al. (1989b) and Brinca et al. (1989).

Daly, P.W. (1987) 'Can Neutral Particles From Comet Halley Have Reached the ICE Spacecraft?', Geophys. Res. Lett, 14, 648.

Erlandson, R.E., L.J. Zanetti, and T.A. Potemra (1989) 'Magnetic-Field Fluctuations from 0 to $26 \mathrm{~Hz}$ Observed From a Polar-Orbiting Satellite,' IEEE Trans. Plasma Science, $17,196$.

European Space Agency, SP-250 (1986) 'The Exploration of Halley's Comet,' ed. B. Battrick, E.J. Rolfe, and R. Reinhard, ESTEC Noordwijk, Holland.

Fairfield, D.H. (1969) 'Bow Shock Associated Wave Observed in the Far Upstream Interplanetary Medium,' J. Geophys. Res., 74, 3541.

Galeev, A.A., R.Z. Sagdeev, V.D. Shapiro, and V.I. Shevchenko (1990) 'MHD Turbulence and Particle Acceleration in a Mass-Loaded Solar Wind,' submitted to J. Geophys. Res.

Gary, S.P., C.W. Smith, M.A. Lee, M.L. Goldstein, and D.W. Forslund (1984) 'Electromagnetic Ion Beam Instabilities,' Phys. Fluids, 27, 1852.

Gary, S.P., C.D. Madland, and B.T. Tsurutani (1985) 'Electromagnetic Ion Beam Instabilities II,' Phys. Fluids, 28, 3691.

Gary, S.P., and C.D. Madland (1988) 'The Electromagnetic Ion Instabilities in a Cometary Environment,' J. Geophys. Res., 93, 235. 
Gary, S.P., C.D. Madland, N. Omidi, and D. Winske (1988) 'Computer Simulation of Two Pickup Ion Instabilities in a Cometary Environment,' J. Geophys. Res., 93, 9584.

Gary, S.P. (1990) 'Electromagnetic Ion/Ion Instabilities and Their Consequences in Space Plasmas: A Review,' Space Science Reviews, in press.

Gary, S.P., K. Akimoto, and D. Winke (1989) 'Computer Simulations of CometaryIon/Ion Instabilities and Wave Growth,' J. Geophys. Res., 94, 3513.

Geophysical Research Letters (1986) ‘Comets I,' 13, 237.

Geophysical Research Letters (1986) 'Comets II,' 13, 381.

Glassmeier, K.H., F.M. Neubauer, M.H. Acuna, and F. Mariani (1987) 'Low-Frequency Magnetic Field Fluctuations in Comet P/Halley's Magnetosheath: Giotto Observations,' Astron. Astrophys. 187, 65.

Glassmeier, K.H., A.J. Coates, M.H. Acuna, M.L. Goldstein, A.D. Johnstone, F.M. Neubauer, and H. Reme (1989) 'Spectral Characteristics of Low-Frequency Plasma Turbulence Upstream of Comet P/Halley,' J. Geophys. Res., 94, 37.

Goldstein, M.L., and H.K. Wong (1987) 'A Theory for Low-Frequency Waves Observed at Comet Giacobini-Zinner,' J. Geophys. Res., 92, 4695.

Goldstein, M.L., H.K. Wong, and K.H. Glassmeier (1990) 'Generation of Low Frequency Waves at Comet Halley,' J. Geophys. Res., 95, 947.

Gosling, J.T., J.R. Ashbridge, S.J. Bame, G. Paschmann, and N. Sckopke (1978) 'Observations of Two Distinct Populations of Bow Shock Ions in the Upstream Solar Wind,' Geophys. Res. Lett. 5, 957.

Grard, R., A. Pedersen, J.-G. Trotignon, C. Beghin, M. Magilevsky, Y. Mikhailov, O. Molchanov, and V. Formisano (1986) 'Observations of Waves and Plasma in the Environment of Comet Halley,' Nature, 321, 290.

Hada, T. (1986) personal communication.

Hada, T., C.F. Kennel, and B. Buti (1989) 'Stationary Nonlinear Alfvén Waves and Solitons,' J. Geophys. Res. 94, 65.

Hasegawa, A. (1969) 'Drift Mirror Instability in the Magnetosphere,' Phys. Fluids, 12, 2642.

Hasegawa, A., (1975) 'Plasma Instabilities and Nonlinear Effects,' Phys. and Chem. in Space, 8, Springer-Verlag, New York, 94.

Hasegawa, A., and L. Chen (1989) 'Theory of the Drift Mirror Instability,' in Plasma Waves and Instabilities at Comets and in Magnetospheres, ed. by B.T. Tsurutani and H. Oya, Geophys, Monogr. Series 54, AGU, Washington, D.C.

Hoppe, M.M., C.T. Russell, L.A. Frank, T.E. Eastman, and E.W. Greenstadt (1981) 'Upstream Hydromagnetic Waves and Their Association With Backstreaming Ion Populations: ISEE 1 and 2 Observations, J. Geophys. Res., 86, 4471.

Hizanidis, K., P.J. Cargill, and K. Papadopoulos (1988) 'Lower Hybrid Waves Upstream of Comets and Their Implications for the Comet Halley "Bow Wave", J. Geophys. Res. 93, 9577.

Hynds, R.J., S.W.H. Cowley, T.R. Sanderson, K.P. Wenzel, and J.J. Van Rooijen, (1986) 'Observations of Energetic Ions From Comet Giacobini-Zinner,' Science, 232, 361.

Ipavich, F.M., A.B. Galvin, G. Gloeckler, D. Hovestadt, B. Klecker, and M. Scholer (1986) 'Comet Giacobini-Zinner: In Situ Observations of Energetic Heavy Ions,' Science, 232, 366. 
Johnstone, A.D., J.A. Bowles, A.J. Coates, A.J. Coker, S.J. Kellock, J. Raymont, B. Wilken, W. Studemann, W. Weiss, R. Cerulli-Irelli, V. Formisano, E. de Giori, P. Perani, M. de Bernardi, H. Borg, S. Olsen, J.D. Winningham, and D.A. Bryant (1976) ESA SP-1077, 15.

Johnstone, A., K. Glassmeier, M. Acuna, H. Borg, D. Bryant, A. Coates, V. Formisano, J. Heath, F. Mariani, G. Musmann, F. Neubauer, M. Thomsen, B. Wilken, and J. Winningham, (1986) Proc. 20th ESLAB Symp., ESA SP-250, Noordwijk, Holland, 277.

Johnstone, A.D., A.J. Coates, J. Heath, M.F. Thomsen, B. Wilken, K. Jockers, V. Formisano, E. Amata, J.D. Winningham, H. Borg, and D.A. Bryant (1987b) 'Alfvénic Turbulence in the Solar Wind Flowing During the Approach to Comet P/Halley,' Astron. Astrophys. 187, 25.

Johnstone, A., K. Glassmeier, M. Acuna, H. Borg, D. Bryant, A. Coates, V. Formisano, J. Heath, F. Mariani, G. Musmann, F. Neubauer, M. Thomsen, B. Wilken, and J. Winningham (1987a) 'Waves in the Magnetic Field and Solar Wind Flow Outside the Bow Shock at Comet P/Halley,' Astron. Astrophys., 187, 47.

Jones, D.E., E.J. Smith, J.A. Slavin, B.T. Tsurutani, G.L. Siscoe, and D.A. Mendis (1986) 'The Bow Wave of Comet Giacobini-Zinner: ICE Magnetic Field Observations, Geophys. Res. Lett., 13, 243.

Kaya, N., H. Matsumoto, and B.T. Tsurutani (1989) 'Test Particle Simulation Study of Whistler Wave Packets Observed Near Comet Giacobini-Zinner,' Geophys. Res. Lett., $16,25$.

Kennel, C.F., and H.E. Petschek (1966) 'Limit on Stable Trapped Particle Fluxes,' J. Geophys. Res., 71, 1.

Kennel, C.F., F.V. Coroniti, F.L. Scarf, B.T. Tsurutani, E.J. Smith, S.J. Bame, and J.T. Gosling (1986) 'Plasma Waves in the Shock Interaction Regions at Comet Giacobini-Zinner,' Geophys. Res. Lett., 13, 921.

Kojima, H., H. Matsumoto, Y. Omura, and B.T. Tsurutani (1989) 'Nonlinear Evolution of High Frequency R-Mode Waves Excited by Water Group Ions Near Comets: Computer Experiments, Geophys. Res., Lett., 16, 9.

Korth, A., A.K. Richter, A. Loidl, K.A. Anderson, C.W. Carlson, D.W. Curtis, R.P. Lin, H. Reme, J.A. Sauvaud, C. d'Uston, F. Cotin, A. Cros, and D.A. Mendis (1986) 'Mass Spectra of Heavy Ions Near Comet Halley,' Nature, 321, 335.

Leckband, J.A., and S.R. Spangler (1989) 'Correlations Between Density and FieldAligned Magnetic Fluctuations in the Earth's Foreshock,' submitted to Geophys. Res. Lett.

Lee, L.C., C.S. Wu, and C.P. Price (1987) 'On The Generation of Magnetosheath Lion Roars,' J. Geophys. Res., 92, 2343.

Lee, M.A. (1989) 'Ultra Low Frequency Waves at Comets,' in Plasma Waves and Instabilities at Comets and in Magnetospheres, ed. B.T. Tsurutani and H. Oya, Am. Geophys. Univ., Washington, D.C., 53, 13.

Lee, M.A., and W.H. Ip (1987) 'Hydromagnetic Wave Excitation by Ionized Interstellar Hydrogen and Helium in the Solar Wind,' J. Geophys. Res. 92, 11041.

Meyer-Vernet, N., P. Couturier, S. Hoang, C. Perche, J.L. Steinberg, J. Fainberg, and C. Meetre (1986) 'Plasma Diagnosis From Thermal Noise and Limits on Dust Flux or Mass in Comet Giacobini-Zinner,' Science, 232, 370. 
Mukai, T., W. Miyake, T. Terasawa, M. Kitayama, and K. Hirao (1986) 'Plasma Observations by Suisei of Solar-Wind Interaction With Comet Halley,' Nature, 321, 299.

Nature (1986) 'Encounters With Comet Halley: The First Results,' 321, 259.

Omidi, N., and D. Winske (1987) 'A Kinetic Study of Solar Wind Mass Loading and Cometary Shocks,' J. Geophys. Res., 92, 13409.

Omidi, N., and D. Winske (1988) 'Subcritical Dispersive Shock Waves Upstream of Planetary Bow Shocks and at Comet Giacobini-Zinner,' Geophys. Res. Lett., 15, 1303.

Omidi, N., and D. Winske (1990) 'Steepening of Kinetic Magnetosonic Waves Into Shocklets: Simulations and Consequences for Planetary Shocks and Comets,' J. Geophys. Res., in press.

Oya, H., A. Morioka, W. Miyake, E.J. Smith, and B.T. Tsurutani (1986) 'Discovery of Cometary Kolmetric Radiation and Plasma Waves at Comet Halley,' Nature, 321, 307.

Price, C.P., D.W. Swift, and L.C. Lee (1986) 'Numerical Simulations of Nonoscillatory Mirror Waves at the Earth's Magnetosheath,' J. Geophys. Res., 91, 101.

Price, C.P., J.D. Gaffey, Jr., and J.Q. Dong (1988) 'Excitation of Low-Frequency Hydromagnetic Waves by Freshly Created Ions in the Solar Wind,' J. Geophys. Res., 93, 837.

Price, C.P. (1989) 'Mirror Waves Driven by Newborn Ion Distributions,' J. Geophys. Res., 94, 15001.

Richardson, I.G., S.W.H. Cowley, R.J. Hynds, P.W. Daly, T.R. Sanderson, and K.P. Wenzel (1989a) 'Properties of Energetic Water Group Ions in the Extended Pick-up Region Surrounding Comet Giacobini-Zinner,' Planet. Space Sci.

Richardson, I.G., K.P. Wenzel, S.W.H. Cowley, F.L. Scarf, E.J. Smith, B.T. Tsurutani, T.R. Sanderson, and R.J. Hynds (1989b) 'Correlated Plasma Wave, Magnetic Field, and Energetic Ion Observations in the Ion Pickup Region of Comet Giacobini-Zinner,' J. Geophys. Res., 94, 49.

Russell, C.T., W. Riedler, K. Schwingenschuh, and Y. Yeroshenko (1987) 'Mirror Instability in the Magnetosphere of Comet Halley,' Geophys. Res. Lett., 14, 644.

Sagdeev, R.Z., V.D. Shapiro, V.I. Shevchenko, and K. Szego (1986) 'MHD Turbulence in the Solar Wind-Comet Interaction Region,' Geophys. Res. Lett., 13, 85.

Sagdeev, R.Z., and A.A. Vedenov (1958) 'Some Properties in a Plasma Having Anisotropic Distribution Function in Magnetic Field,' in Plasma Physics and Controlled Fusion, M.A. Leontovich, Atomizdat, Moscow, 3, 278.

Saito, T., K. Yumoto, K. Hirao, T. Nakagawa, and K. Saito (1986) 'Interaction Between Comet Halley and the Interplanetary Magnetic Field Observed by Sakigake,' Nature, $321,303$.

Sanderson, T.R., K.-P. Wenzel, P. Daly, S.W.H. Cowley, R.J. Hynds, E.J. Smith, S.J. Bame, and R.D. Zwickl (1986) 'The Interaction of Heavy Ions From Comet P/Giacobini-Zinner With the Solar Wind,' Geophys. Res. Lett., 13, 411.

Scarf, F.L., R.W. Fredericks, D.A. Gurnett, and E.J. Smith (1978) 'ISEE-C Plasma Wave Instrument,' IEEE Trans. Geosci. Electron., GE-16, 225.

Scarf, F.L., F.V. Coroniti, C.F. Kennel, T.R. Sanderson, K.-P. Wenzel, R.J. Hynds, E.J. Smith, S.J. Bame, and R.D. Zwickl (1986a) 'ICE Plasma Wave Measurements in the Ion Pickup Region of Comet Halley,' Geophys. Res. Lett., 13, 857. 
Scarf, F.L., F.V. Coroniti, C.F. Kennel, D.A. Gurnett, W.-H. Ip, and E.J. Smith (1986b) 'Plasma Wave Observations at Comet Giacobini-Zinner,' Science, 232, 377.

Scarf, F.L. (1989) 'Plasma Wave Observations at Comets Giacobini-Zinner and Halley,' in Plasma Waves and Instabilities at Comets and in Magnetospheres, ed. by B.T.

Tsurutani and H. Oya, Amer. Geophys. Un., Washington, D.C., 53, 31.

Science (1986) 'The International Cometary Explorer Mission to Comet Giacobini-Zinner,' $232,353$.

Sentman, D.D., J.P. Edmiston and L.A. Frank (1981) 'Instabilities of Low Frequency,' Parallel Propagating Electromagnetic Waves in the Earth's Foreshock Region,' J.

Geophys. Res., 86, 7487.

Shapiro, V.D., and V.I. Shevchenko (1988) 'Astrophysical Plasma Turbulence in Soviet

Science Reviews,' Section E, ed. by R.A. Syunyaev, Harwood Acad. Pub., London, England, 427.

Sharma, A.S., P.J. Cargill and K. Papadopoulos (1988) 'Resonance Absorption of Alfvén

Waves at Comet-Solar Wind Interaction Regions,' Geophys. Res. Lett., 15, 74.

Sharma, O.P. and V.L. Patel, (1986) 'Low-Frequency Electromagnetic Waves Driven by

Gyrotropic Gyrating Ion Beams, J. Geophys. Res., 91, 1529,

Siscoe, G.L., L. Davis, Jr., P.J. Coleman, Jr., E.J. Smith and D.E. Jones (1968) 'Power

Spectra and Discontinuities of the Interplanetary Magnetic Field: Mariner 4,'J.

Geophys. Res., 73, 61.

Slavin, J.A., E.J. Smith, and B.T. Tsurutani (1987) 'Magnetic Field Structures in the

Comet Giacobini-Zinner Ionosheath,' paper presented at 19th General Assembly,

IUGG, Vancouver, B.C.

Smith, C.W., M.L. Goldstein, S.P. Gary, and C.T. Russell (1985) 'Beam Driven Ion

Cyclotron Harmonic Resonance in the Terrestrial Foreshock,' J. Geophys. Res., 90, 1429.

Smith, C.W., and S.P. Gary (1987) 'Electromagnetic Instabilities: Growth at Cyclotron Harmonic Wave Numbers,' J. Geophys. Res., 92, 117.

Smith, E.J., B.T. Tsurutani, J.A. Slavin, D.E. Jones, G.L. Siscoe, and D.A. Mendis (1986a) 'International Cometary Explorer Encounter with Giacobini-Zinner: Magnetic Field Observations, Science, 232, 382.

Smith, E.J., J.A. Slavin, S.J. Bame, M.F. Thomsen, S.W.H. Cowley, I.G. Richardson, D. Hovestadt, F.M. Ipavich, K.W. Ogilvie, M.A. Coplan, T.R. Sanderson, K.P.

Wenzel, F.L. Scarf, A.F. Vinas, and J.D. Scudder (1986b) 'Analysis of the GiacobiniZinner Bow Wave,' Proc. 20th ESLAB Symp. Explor. Halley's Comet, ESA SP-250, 461.

Smith, E.J., B.T. Tsurutani, J.A. Slavin, F.L. Scarf, and S.J. Bame (1987) 'Waves in the Giacobini-Zinner Magnetosheath: ICE Observations,' in Proc. Chapman Conference on Plasma Waves and Instabilities in Magnetosphere and at Comets, ed. $\mathrm{H}$. Oya and B.T. Tsurutani, Sohbun Insatsu, Sendai, Japan.

Somogyi, A.J., K.I. Grimgauz, K. Szego et al. (1986) 'First Observations of Energetic

Particles Near Comet Halley,' Nature, 321, 285.

Spangler, S., S. Fuselier, A. Fey, and G. Anderson (1988) 'An Observational Study of MHD Wave-Induced Density Fluctuations Upstream of the Earth's Bow Shock,' J.

Geophys. Res., 93, 845. 
Terasawa, T. (1989) 'Particle Scattering and Acceleration in a Turbulent Plasma Around Comets,' in Plasma Waves and Instabilities at Comets and in Magnetospheres, ed. B.T. Tsurutani and H. Oya, Am. Geophys. Un., 53, 41.

Thorne, R.M., and B.T. Tsurutani (1987) 'Resonant Interactions Between Cometary Ions and Low Frequency Electromagnetic Waves,' Planet. Space Sci., 35, 1501.

Tranquille, C., I.G. Richardson, S.W.H. Cowley, T.R. Sanderson, K.-P. Wenzel, and R.J. Hynds (1986) 'Energetic Ion Properties Observed Near the Periphery of the MassLoaded Flow Region Surrounding Comet P/Giacobini-Zinner,' Geophys. Res. Lett., $13,853$.

Tsurutani, B.T., E.J. Smith, R.R. Anderson, K.W. Ogilvie, J.D. Scudder, D.N. Baker, and S.J. Bame (1982) 'Lion Roars and Nonoscillatory Drift Mirror Waves in the Magnetosheath,' J. Geophys. Res., 87, 6060.

Tsurutani, B.T., and E.J. Smith (1986a).'Hydromagnetic Waves and Instabilities Associated With Cometary-Ion Pickup: ICE Observations,' Geophys. Res. Lett., 13, 263.

Tsurutani, B.T., and E.J. Smith (1986b) 'Strong Hydromagnetic Turbulence Associated With Comet Giacobini-Zinner,' Geophys. Res. Lett., 13, 259.

Tsurutani, B.T., A.L. Brinca, E.J. Smith, R.M. Thorne, F.L. Scarf, J.T. Gosling, and F.M. Ipavich (1987a) 'MHD Waves Detected by ICE at Distance $\geq 28 \times 10^{6} \mathrm{~km}$ From Comet P/Halley: Cometary or Solar Wind Origin?', Astron. Astrophys., 187, 97.

Tsurutani, B.T., R.M. Thorne, E.J. Smith, J.T. Gosling, and H. Matsumoto (1987b) 'Steepened Magnetosonic Waves at Comet Giacobini-Zinner,' J. Geophys. Res., 92, 11074.

Tsurutani, B.T., D.E. Page, E.J. Smith, B.E. Goldstein, A.L. Brinca, R.M. Thorne, H. Matsumoto, I.G. Richardson, and T.R. Sanderson (1989a) 'Low-Frequency Plasma Waves and Ion Pitch Angle Scattering at Large Distances $\left(>3.5 \times 10^{5} \mathrm{~km}\right)$ From Giacobini-Zinner: Interplanetary Magnetic Field $\alpha$ Dependence,' J. Geophys. Res., $94,18$.

Tsurutani, B.T., A.L. Brinca, B. Buti, E.J. Smith, R.M. Thorne, and H. Matsumoto (1989b) 'Magnetic Pulses With Durations Near The Local Proton Cyclotron Period: Comet Giacobini-Zinner,' J. Geophys. Res., 94, 29.

Tsurutani, B.T., E.J. Smith, A.L. Brinca, R.M. Thorne, and H. Matsumoto (1989c)

'Properties of Whistler Mode Wave Packets at the Leading Edge of Steepened Magnetosonic Waves: Comet Giacobini-Zinner,' Planet. Space Sci., 37, 167.

Tsurutani, B.T., and E.J. Smith (1989) 'New Nonlinear Comet Giacobini-Zinner Wave Features: ICE Observations,' EOS, 70, 1181.

Wenzel, K.-P., T.R. Sanderson, I.G. Richardson, S.W.H. Cowley, R.J. Hynds, S.J. Bame, R.D. Zwickl, E.J. Smith, and B.T. Tsurutani (1986) 'In-Situ Observations of Cometary Pick-Up Ions $\geq 0.2$ AU Upstream of Comet Halley: ICE Observations,' Geophys. Res. Lett., 13, 861.

Winske, D., C.S. Wu, Y.Y. Li, Z.Z. Mou, and S.Y. Gao (1985) 'Coupling of Newborn Ions to the Solar Wind by Electromagnetic Instabilities and the Interaction With the Bow Shock,' J. Geophys. Res., 90, 2713.

Winske, D., and S.P. Gary (1986) 'Electromagnetic Instabilities Driven by Cool Heavy Ion Beams,' J. Geophys. Res., 91, 6825. 
Winske, D., and M.M. LeRoy (1984) 'Diffuse Ions Produced by Electromagnetic Ion Beam Instabilities,' J. Geophys. Res., 89, 2673.

Wu, C.S., and R.C. Davidson (1972) 'Electromagnetic Instabilities Produced by Neutral Particle Ionization in Interplanetary Space,' J. Geophys. Res. 72, 5399.

Wu, C.S., and R.E. Hartle (1974) 'Further Remarks on Plasma Instabilities Produced by Ions Borne in the Solar Wind,' J. Geophys. Res., 79, 283.

Yeroshenko, Y.G., V.A. Styashkin, W. Riedler, K. Schwingenschuh, and C.T. Russell (1987) Fine Structure of the Magnetic Field on Comet P/Halley's Comet,' Astron. Astrophys., 187, 69. 